\title{
Cinobufotalin as an effective adjuvant therapy for advanced gastric cancer: a meta-analysis of randomized controlled trials
}

This article was published in the following Dove Press journal: OncoTargets and Therapy

\author{
Huiling Sun' \\ Wenxiao Wang ${ }^{2}$ \\ Minghua $\mathrm{Bai}^{3}$ \\ Dongling $\mathrm{Liu}^{4}$
}

'Department of Gastroenterology, Liaocheng People's Hospital, Liaocheng, Shandong 252000, People's Republic of China; ${ }^{2}$ Department of

Gastroenterological Surgery, Liaocheng People's Hospital, Liaocheng, Shandong 252000, People's Republic of China; ${ }^{3}$ Department of Health, Liaocheng People's Hospital, Liaocheng, Shandong 252000, People's Republic of China; ${ }^{4}$ Department of Pharmacy, Liaocheng People's Hospital, Liaocheng, Shandong 252000, People's Republic of China
Correspondence: Dongling Liu Department of Pharmacy, Liaocheng People's Hospital, Dongchang West Road, No.67, Liaocheng, Shandong 252000,

People's Republic of China

Tel +86 I3780720620

Email dongling162@।63.com
Purpose: This study aimed to investigate the efficacy and safety of combining cinobufotalin and chemotherapy for advanced gastric cancer (GC).

Patients and methods: Literature retrieval was performed in Cochrane Library, Web of Science, PubMed, Embase, China National Knowledge Infrastructure (CNKI), Chinese Biological Medicine Database (CBM), Wanfang database and Chinese Scientific Journal Database (VIP) before September 2018. The primary reported outcomes including therapeutic efficacy, quality of life (QoL), and adverse events were systematically evaluated.

Results: Data from 27 trials including 1,939 advanced GC patients were included. The results indicated that, compared with chemotherapy alone, the combination of chemotherapy and cinobufotalin significantly improved patients' overall response rate (odds ratio [OR] $=1.88,95 \%$ confidence interval $[\mathrm{CI}]=1.54-2.31, P<0.00001)$ and disease control rate $(\mathrm{OR}$ $=2.05,95 \% \mathrm{CI}=1.63-2.58, P<0.00001)$. The QoL of patients also evidently improved after chemotherapy and cinobufotalin combined treatment, as indicated by increased QoL improved rate $(\mathrm{OR}=2.39,95 \% \mathrm{CI}=1.81-3.15, P<0.00001)$, Karnofsky Performance Score $(\mathrm{OR}=7.00,95 \% \mathrm{CI}=2.25-11.75, P=0.004)$ and pain relief rate $(\mathrm{OR}=7.00,95 \% \mathrm{CI}=2.25-$ 11.75, $P=0.004)$. Adverse events including nausea and vomiting, diarrhea, leukopenia, handfoot syndrome, anemia, gastrointestinal side effects and peripheral neurotoxicity caused by chemotherapy were evidently alleviated $(P<0.05)$ when cinobufotalin was administered to GC patients.

Conclusion: Evidence from the meta-analysis suggested that the combination of chemotherapy and cinobufotalin is more effective in treating GC than chemotherapy alone. It alleviates the adverse effects associated with chemotherapy and improves the QoL of GC patients.

Keywords: cinobufotalin, traditional Chinese medicine, chemotherapy, gastric cancer, metaanalysis

\section{Introduction}

Gastric cancer (GC) represents the second leading cause of death among all cancer types and caused 782,685 deaths worldwide in 2018. ${ }^{1}$ Currently, the incidence of GC has significantly increased, with about $1,033,701$ new cases every year. ${ }^{1}$ China has a high risk for GC, and the new cases of GC in this region account for about $43 \%$ in the world. ${ }^{2}$ Despite the improvement of diagnostic and therapeutic methods in the past decades, ${ }^{3,4}$ the prognosis of GC is still poor ( 5 -year survival rate $<20 \%$ ) since it is mostly diagnosed at advanced stage. ${ }^{3,4}$ Therefore, effective therapeutic approaches should be developed. 
Traditional Chinese medicine has an extensive history and has been more widely used as an effective adjuvant drug for cancer treatment. ${ }^{5-10}$ Cinobufotalin is a cardiotonic steroid or bufotalin, which is extracted from the skin secretion of the giant toad. ${ }^{10-14}$ Many in vitro studies have shown that cinobufotalin has antitumor activity and enhanced chemotherapeutic effect. ${ }^{7,10,13,14}$ Cinobufotalin can inhibit the growth and metastasis of the tumor by inhibiting the expression of vascular endothelial growth factor and epidermal growth factor receptor. ${ }^{15}$ Additionally, it can also kill tumor cells by inducing nonapoptotic death possibly depending on cyclophilin-D involved pathway. ${ }^{12}$

Several studies have indicated that chemotherapy combined with cinobufotalin exhibits more prominent therapeutic effects than chemotherapy alone for advanced GC. ${ }^{16-42}$ Despite the intensive clinical studies using cinobufotalin and chemotherapy combined therapy in treating GC, its clinical efficacy and safety have not been systematically evaluated. In this study, we conducted a metaanalysis to investigate the treatment efficacy and safety of chemotherapy combined with cinobufotalin in comparison with chemotherapy alone for advanced GC to provide scientific reference for the design of future clinical trials.

\section{Materials and methods}

\section{Search strategy and selection criteria}

This meta-analysis was performed in accordance with the Preferred Reporting Items for Systematic Reviews and MetaAnalyses (PRISMA) guidelines and Cochrane Handbook. Original articles were searched across eight electronic databases, including Cochrane Library, Web of Science, PubMed, Embase, China National Knowledge Infrastructure (CNKI), Chinese Biological Medicine Database (CBM), Wanfang database and Chinese Scientific Journal Database (VIP) before September 2018, with key terms "huachansu" or "cinobufotalin," "cinobufacini," or "cinobufagin" combined with "gastric carcinoma" or "gastric cancer." No language limits were applied.

Selection Criteria: The inclusion criteria were as follows: (1) controlled trials concerning advanced GC patients, (2) literature comparing the clinical outcomes of chemotherapy plus cinobufotalin adjuvant therapy (experimental group) with chemotherapy treatments alone (control group) and (3) articles involving more than $40 \mathrm{GC}$ patients. On the other hand, the exclusion criteria were as follows: (1) non-contrast articles, case studies and review papers and (2) patients with mixed malignancies.

\section{Data extraction and quality assessment}

Data were independently extracted by two investigators (Sun HL, and Bai MH) following the same inclusion criteria; disagreements were adjudicated by the third reviewer (Liu DL). The extracted characteristics were summarized as follows: (I) first author's names, (II) years of publication, (III) study locations, (IV) tumor stages, (V) Karnofsky Performance Score (KPS), (VI) number of cases, (VII) patient ages, (VIII) study parameter types, (IX) therapeutic regimens, (X) enrollment period and (XI) dosage of cinobufotalin. The included trial's quality was evaluated according to the Cochrane Handbook. ${ }^{43}$

\section{Outcome definition}

Clinical responses include treatment efficacy, quality of life (QoL) and adverse events. Treatment efficacy was assessed in terms of the overall survival rates (OS rates, defined as the length of time from the start of treatment to death from any cause), complete response (CR) rates, partial response (PR) rates, stable disease $(\mathrm{SD})$ rates, progressive disease $(\mathrm{PD})$ rates, overall response rates $(\mathrm{ORRs}, \mathrm{ORR}=\mathrm{CR}+\mathrm{PR})$ and disease control rates (DCRs, $\mathrm{DCR}=\mathrm{CR}+\mathrm{PR}+\mathrm{SD}$ ). Patients' QoL was evaluated using QoL improved rate (QIR), KPS and pain relief rate (PRR). Adverse events including nausea and vomiting, diarrhea, leucopenia, thrombocytopenia, hepatotoxicity, nephrotoxicity, oral mucositis, alopecia, hand-foot syndrome, anemia, gastrointestinal adverse effects, peripheral neurotoxicity, neutropenia and myelosuppression were also assessed.

\section{Statistical analysis}

RevMan 5.3 (Nordic Cochran Centre, Copenhagen, Denmark) and Stata 13.0 (Stata Corp., College Station, TX, USA) software were the main statistical analysis tools in this study. $P<0.05$ was considered statistically significant. Analysis model was determined by heterogeneity among studies assessed using Cochran's Q test, and publication bias was analyzed using Begg's and Egger's regression asymmetry tests and presented using funnel plots. ${ }^{44} I^{2}<50 \%$ or $P>0.1$ indicated that the studies were homogenous. Treatment effects were mainly represented by odds ratio (OR) presented with a $95 \%$ confidence 
interval (CI). Pooled analysis with publication bias determined that trim and fill method would be applied to coordinate the estimates of unpublished studies, and the adjusted results were compared with the original pooled OR. ${ }^{45}$ Sensitivity analysis was performed to evaluate the impact of different therapeutic regimens, drug forms of cinobufotalin, sample sizes and research types on clinical efficacy.

\section{Results}

\section{Search results}

A total of 493 articles were identified and initially retrieved, and 275 papers were excluded due to duplication. After title and abstract review, 163 articles were further excluded because they did not include clinical trials $(n=127)$ and were unrelated studies $(n=34)$ or published before $2000(n=2)$, leaving 55 studies as potentially relevant. After detailed assessment of full texts, articles without control group $(n=8)$, studies with case reports $(n=6)$, reviews or meta-analysis $(n=5)$, and trials with insufficient data $(n=9)$ were excluded. Finally, 27 trials $^{16-42}$ involving 1,939 advanced GC patients were included in this analysis (Figure 1).

\section{Patient characteristics}

After selection, all included studies were performed in different medical centers of China since 2000. In total, 972 advanced GC patients were treated with chemotherapy in combination with cinobufotalin adjuvant therapy, while 967 patients were treated with chemotherapy alone. Detailed information of the involved studies and GC patients is shown in Tables 1 and 2 .

\section{Quality assessment}

The assessment of bias risk is shown in Figure 2. A total of 24 studies were determined as having low risk, and the remaining 3 studies were not true randomized controlled

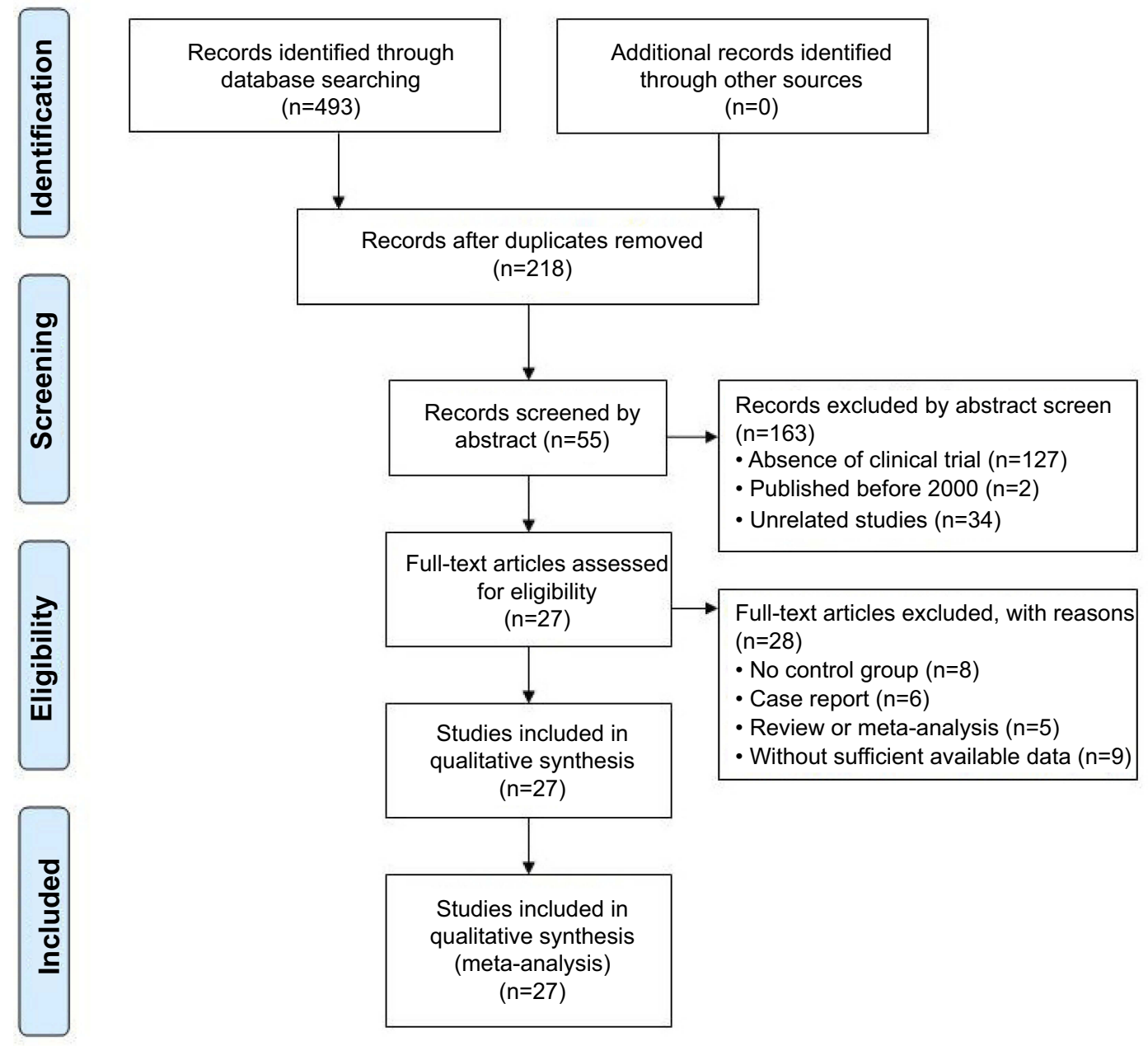

Figure I Flow diagram of the selection process. 


\begin{tabular}{|c|c|}
\hline & 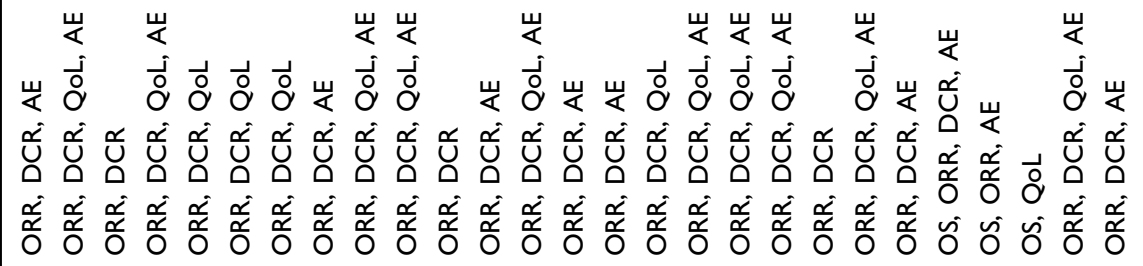 \\
\hline 妾 & 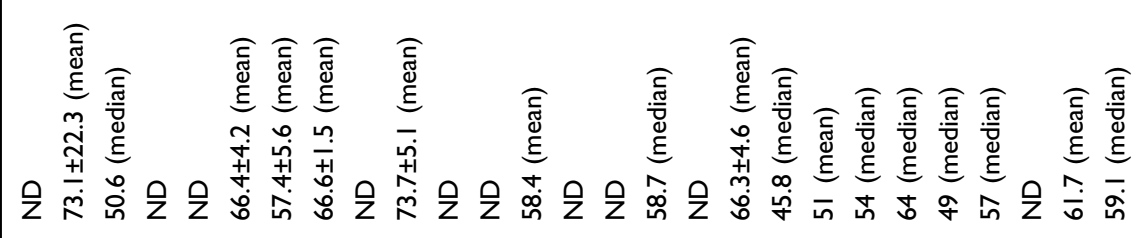 \\
\hline نे & 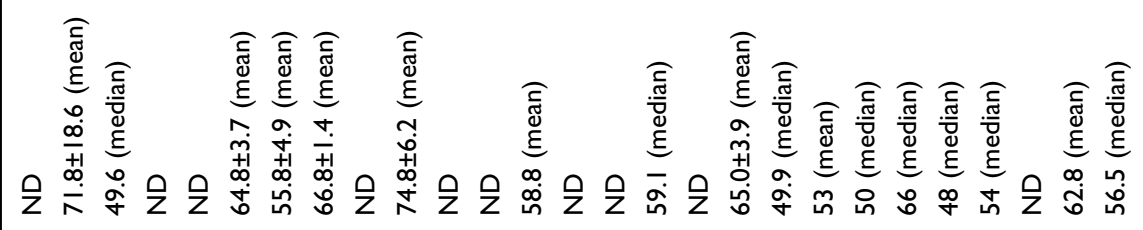 \\
\hline & 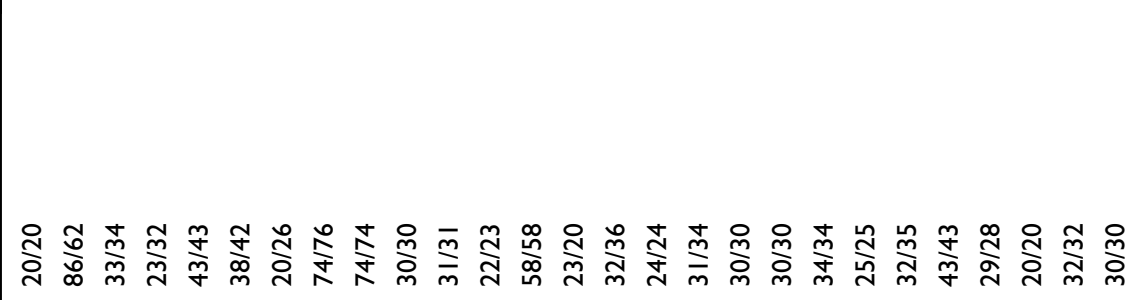 \\
\hline & 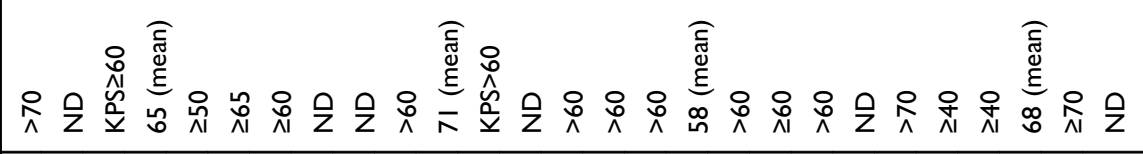 \\
\hline & 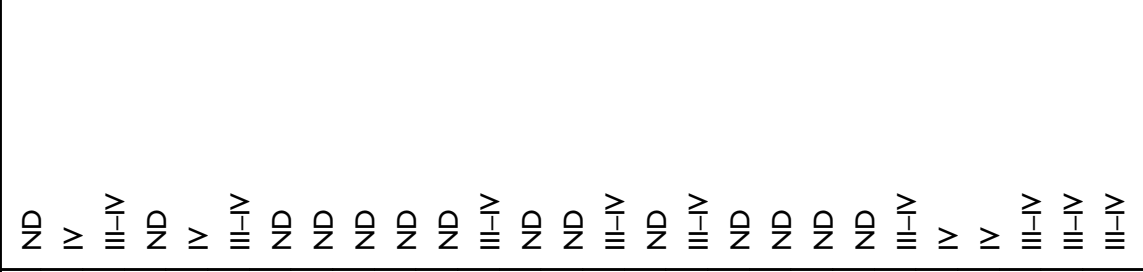 \\
\hline & 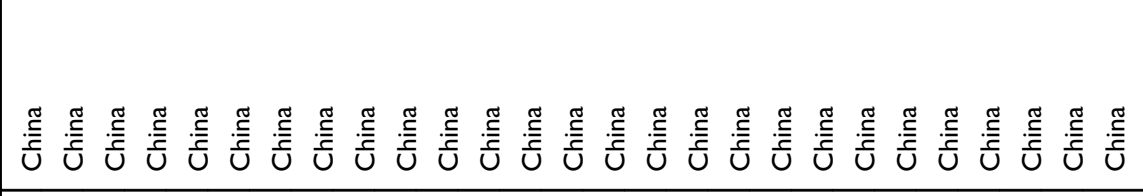 \\
\hline & 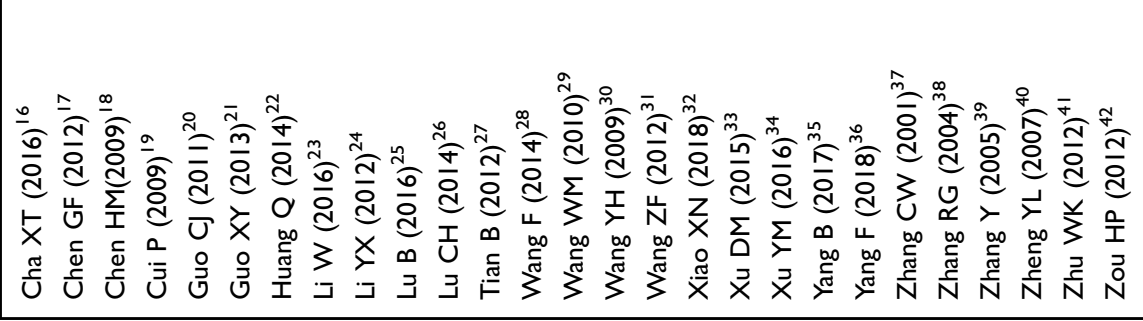 \\
\hline
\end{tabular}




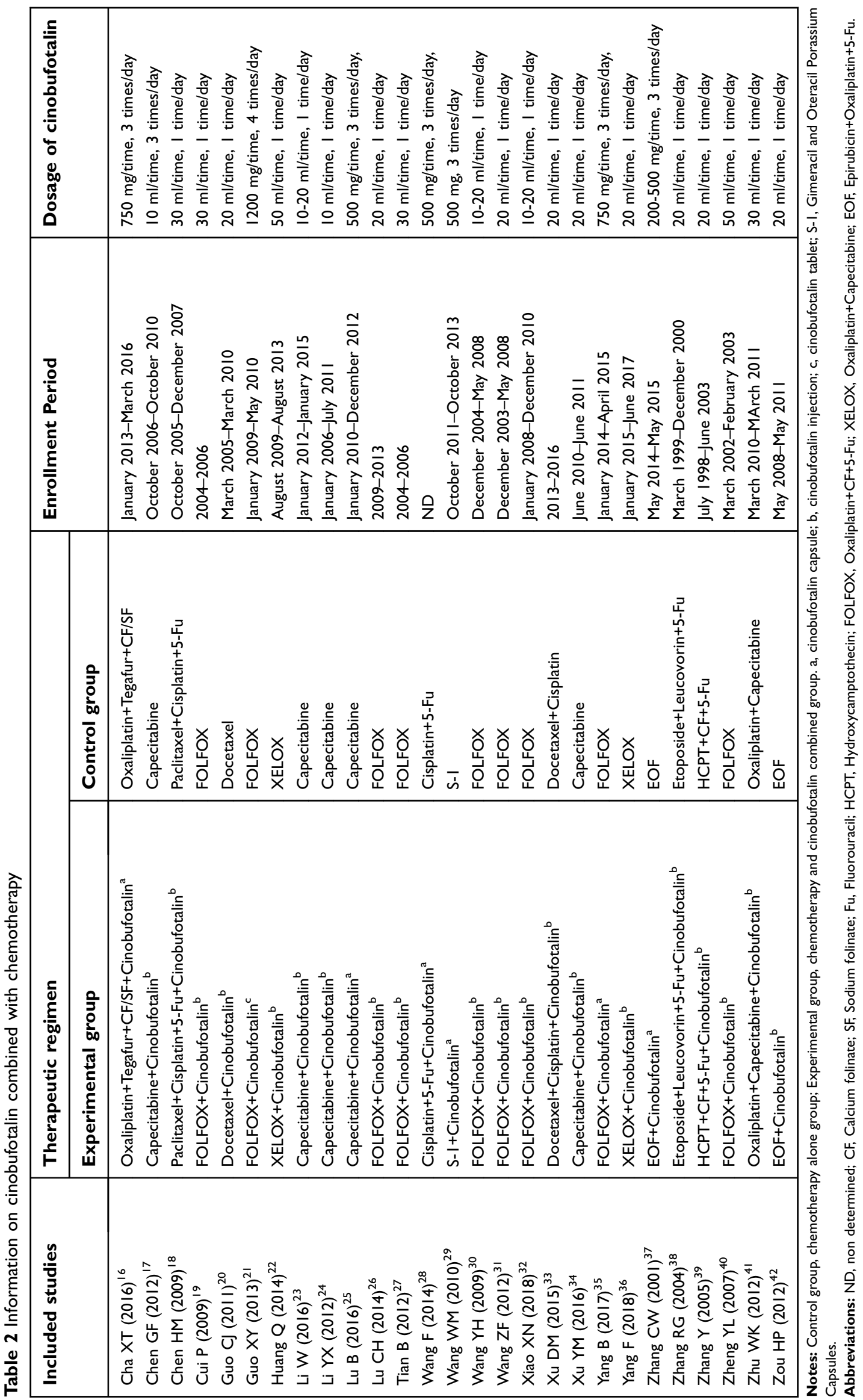


A

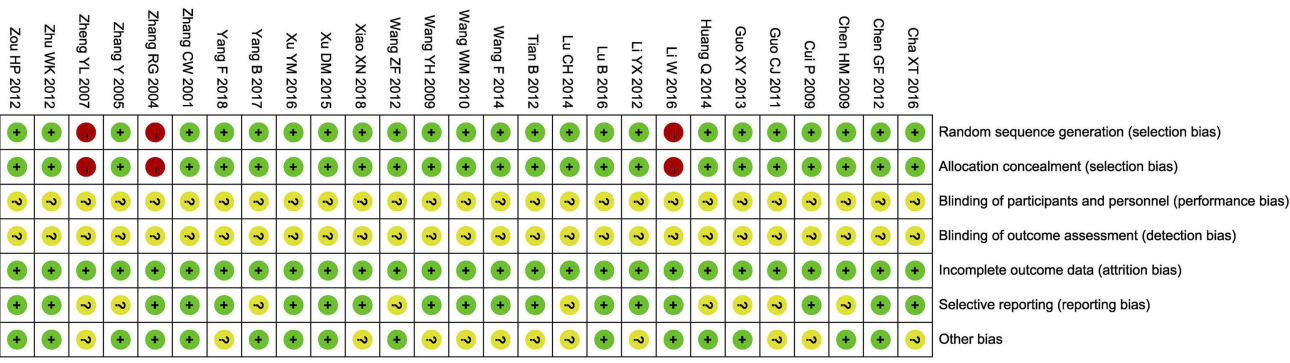

B

Random sequence generation (selection bias) Allocation concealment (selection bias) Blinding of participants and personnel (performance bias) Blinding of outcome assessment (detection bias) Incomplete outcome data (attrition bias) Selective reporting (reporting bias)

Other bias

Low risk of bias

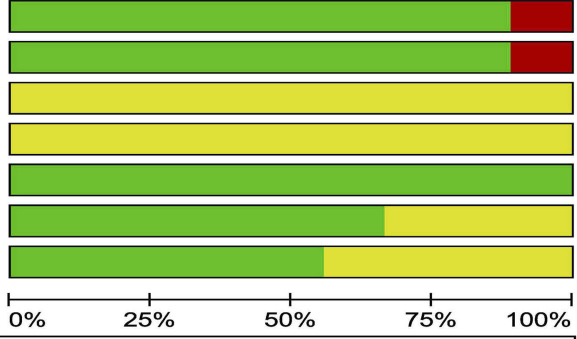

$\begin{array}{lll}0 \% & 25 \% & 50 \% \\ & \text { High risk of bias }\end{array}$

Figure 2 (A) Risk of bias summary: review of authors' judgments about each risk of bias item for included studies. (B) Risk of bias graph: review of authors' judgments about each risk of bias item presented as percentages across all included studies. Each color represents a different level of bias: red for high-risk, green for low-risk and yellow for unclear-risk of bias.

trials. All included trials did not provide clear description of performance and detection risks. The attrition risks of involved trials were low; 9 trials were considered as having unclear risk owing to selective reporting.

\section{Therapeutic efficacy assessment}

As shown in Figures 3 and 4, Figure S1 and Table 3, the pooled results showed that patients who underwent combined therapy had significantly improved CR, PR, ORR and DCR (CR, OR $=1.69,95 \%$ CI $=1.11-2.57$, $P=0.01 ; \quad$ PR, $\quad$ OR $=1.69, \quad 95 \% \quad$ CI $=1.38-2.08$, $P<0.00001 ;$ ORR, OR $=1.88,95 \%$ CI $=1.54-2.31$, $P<0.00001 ;$ DCR, OR $=2.05,95 \%$ CI $=1.63-2.58$, $P<0.00001$ ) and significantly decreased PD (OR $=0.49,95 \%$ CI $=0.39-0.61, P<0.00001)$, whereas SD and 6- and 12-months OS rates had no significant

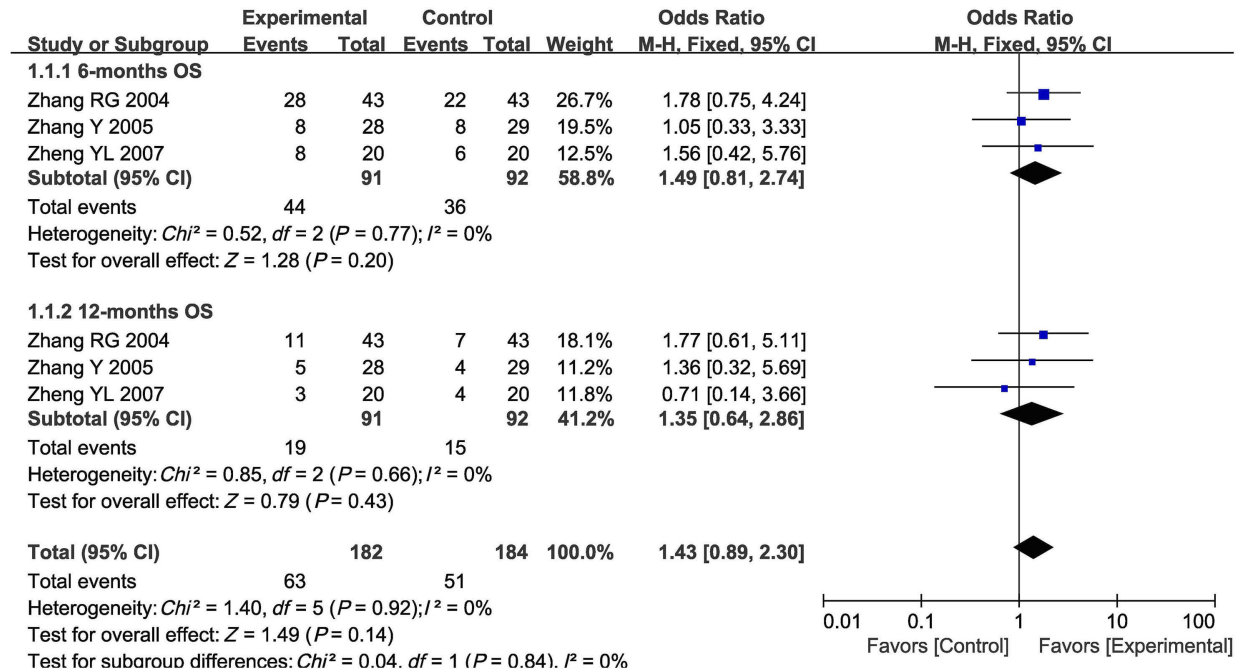

Figure 3 Forest plot of the comparison of 6-months (A) and I2-months (B) overall survival (OS) between the experimental and control group. Control group, chemotherapy alone group; Experimental group, chemotherapy and cinobufotalin combined group. The fixed-effects meta-analysis model (Mantel-Haenszel method) was used. 
A

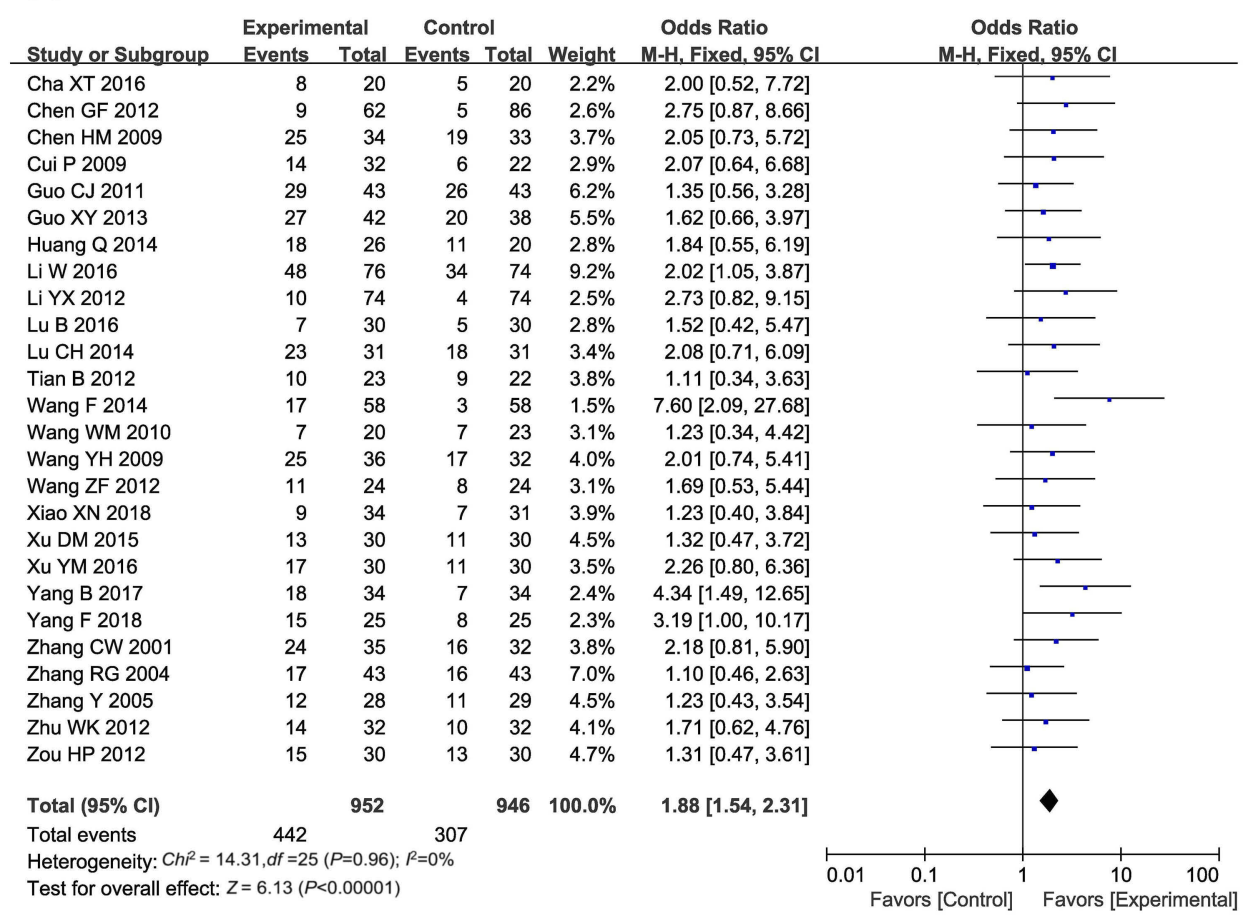

\section{B}

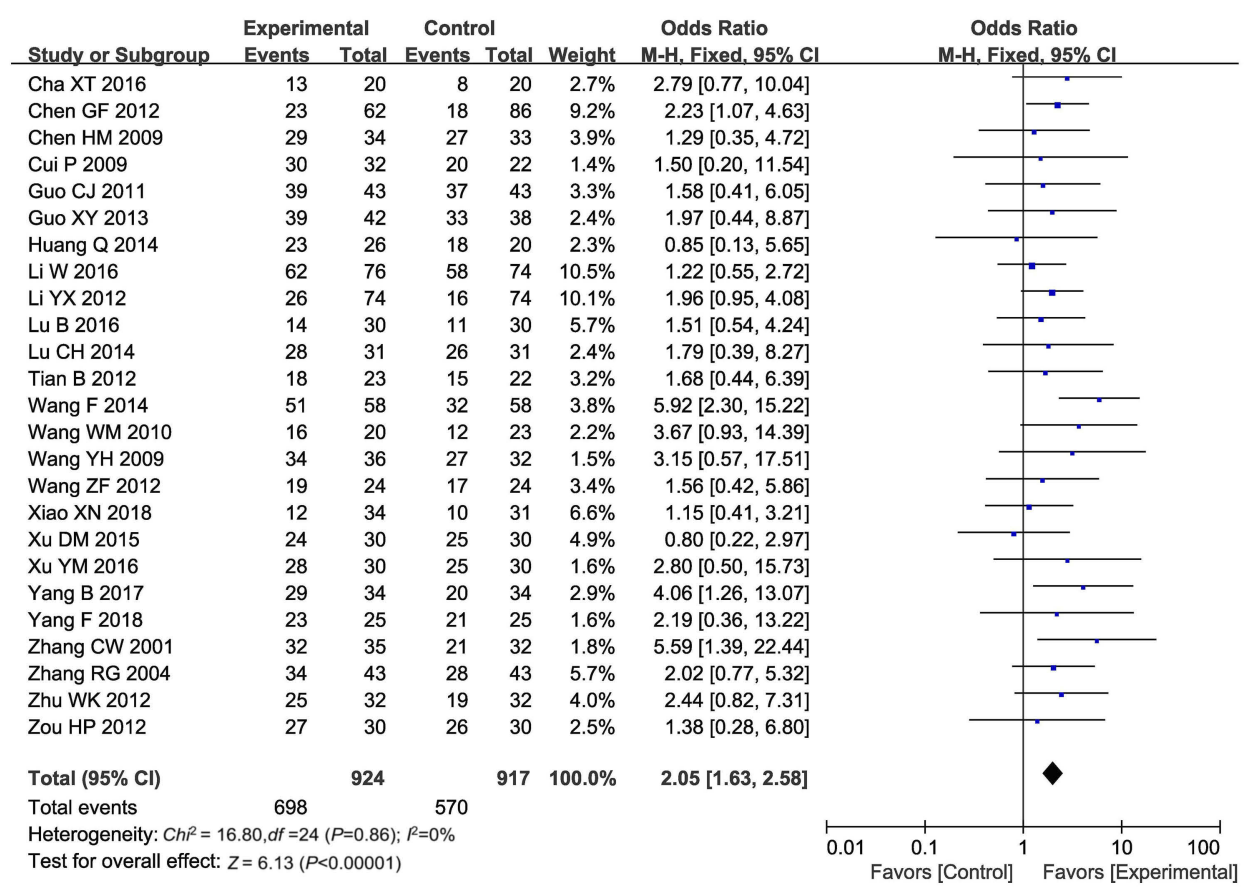

Figure 4 Forest plot of the comparison of overall response rate (ORR, A) and disease control rate (DCR, B) between the experimental and control group. Control group, chemotherapy alone group; Experimental group, chemotherapy and cinobufotalin combined group. The fixed-effects meta-analysis model (Mantel-Haenszel method) was used.

differences in patients who received chemotherapy alone $(\mathrm{SD}, \mathrm{OR}=0.94,95 \% \mathrm{CI}=0.76-1.15, P=0.53 ; 6$ months OS, OR $=1.49,95 \% \mathrm{CI}=0.81-2.74, P=0.20$; 12-months OS, OR $=1.35,95 \%$ CI $=0.64-2.86$, $P=0.43$ ). Fixed effect models were used to analyze OR rate because of low heterogeneity. 


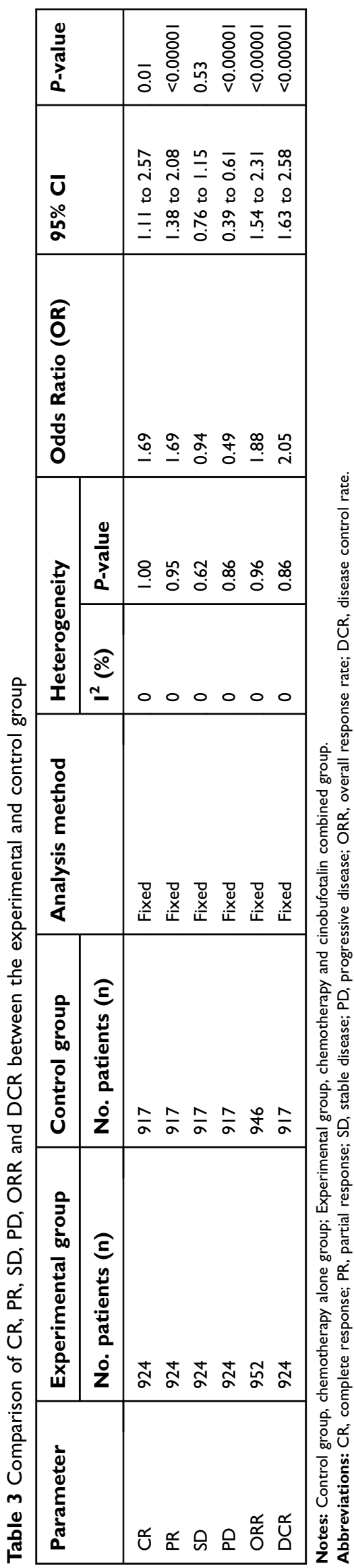

\section{Quality of life assessment}

QoL was evaluated in this analysis. Result showed that QoL of patients in the combined group was significantly better than that of the control group, indicated by increased QIR, KPS and PRR (Figure 5, QIR, OR $=2.39,95 \%$ CI $=1.81-3.15, P<0.00001$; KPS, OR $=7.00,95 \% \mathrm{CI}=2.25$ 11.75, $P=0.004$; PRR, OR $=4.06,95 \%$ CI $=2.24-7.35$, $P<0.00001)$.

\section{Adverse event assessment}

As shown in Table 4 and Figure S2, patients treated with cinobufotalin and chemotherapy combined therapy showed lower incidences of nausea and vomiting, diarrhea, leucopenia, hand-foot syndrome, anemia, gastrointestinal side effects and peripheral neurotoxicity (nausea and vomiting, $\mathrm{OR}=0.55$, $95 \% \mathrm{CI}=0.41-0.74, P<0.0001$; diarrhea, $\mathrm{OR}=0.65,95 \% \mathrm{CI}$ $=0.46-0.90, P=0.010$; leucopenia, $\mathrm{OR}=0.62,95 \% \mathrm{CI}=0.47-$ 0.82, $P=0.0008$; hand-foot syndrome, $\mathrm{OR}=0.57,95 \% \mathrm{CI}$ $=0.41-0.79, P=0.0007$; anemia, $\mathrm{OR}=0.69,95 \% \mathrm{CI}=0.48$ $0.99, P=0.05$; gastrointestinal side effects, $\mathrm{OR}=0.56,95 \% \mathrm{CI}$ $=0.32-1.00, P=0.05$; peripheral neurotoxicity, $\mathrm{OR}=0.32,95 \%$ $\mathrm{CI}=0.20-0.50, P<0.00001$ ), whereas analysis on thrombocytopenia, hepatotoxicity, nephrotoxicity, oral mucositis, alopecia, neutropenia and myelosuppression (thrombocytopenia, $\mathrm{OR}=0.69,95 \% \mathrm{CI}=0.44-1.11, P=0.13$; hepatotoxicity, $\mathrm{OR}$ $=0.53,95 \% \mathrm{CI}=0.24-1.16, P=0.11$; nephrotoxicity, $\mathrm{OR}=0.56$, $95 \% \mathrm{CI}=0.16-1.95, P=0.36$; oral mucositis, $\mathrm{OR}=0.62,95 \%$ $\mathrm{CI}=0.28-1.34, P=0.22$; alopecia, $\mathrm{OR}=0.61,95 \% \mathrm{CI}=0.24$ $1.56, P=0.30$; neutropenia, $\mathrm{OR}=0.45,95 \% \mathrm{CI}=0.14-1.42$, $P=0.17$; myelosuppression, $\mathrm{OR}=0.38,95 \% \mathrm{CI}=0.08-1.84$, $P=0.23$ ) did not differ significantly between the two groups.

\section{Publication bias}

Funnel plots drawn for the studies on primary outcomes (CR, PR, SD, PD, ORR, DCR and adverse events) were approximately symmetrical, which indicated generally controlled publication bias and reliability of our primary conclusions (Figure 6 and S3).

We also assessed publication bias using Begg's and Egger's regression asymmetry tests (Table 5), and PR and leucopenia were found with bias (PR, Begg, 0.038; Egger, 0.015; leucopenia, Begg, 0.003; Egger, <0.0001). To determine if the bias affects the pooled risk, we conducted a trim and fill analysis. The adjusted OR rate indicated the same trend with the result of the primary analysis (PR [before, $P<0.0001$; after, $P<0.0001$ ], leukopenia [before, $P=0.0002$; after. $P=0.0002]$ ), reflecting the 
A

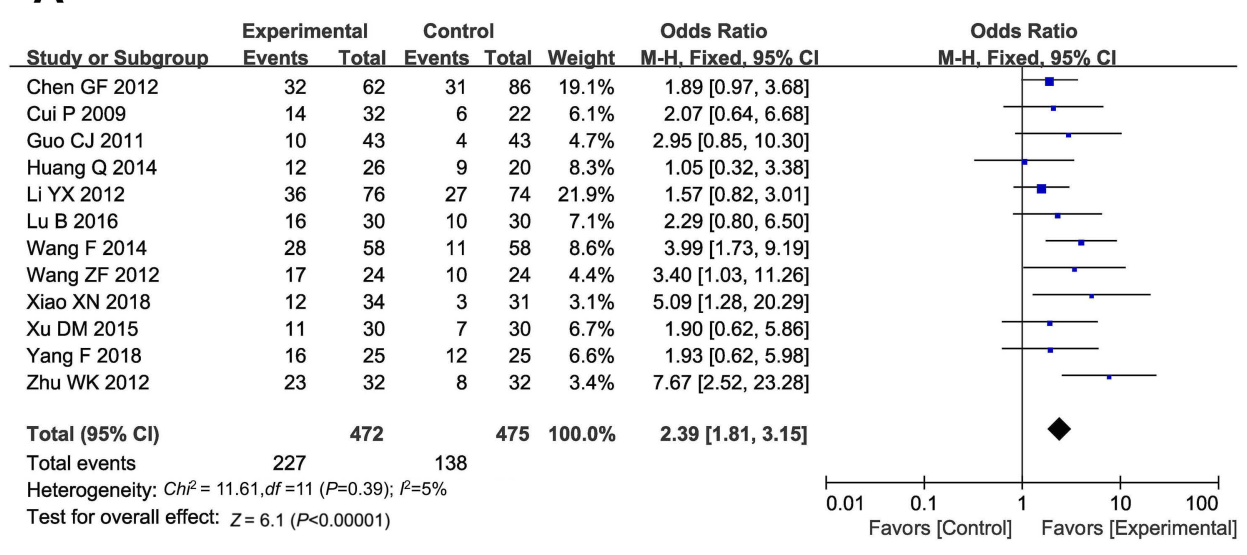

B

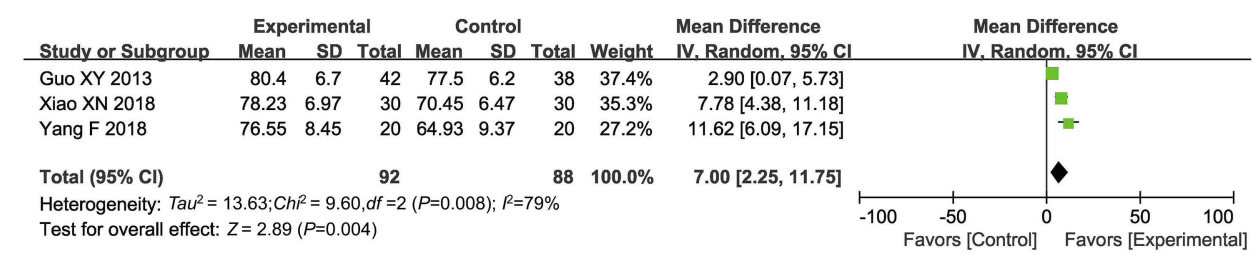

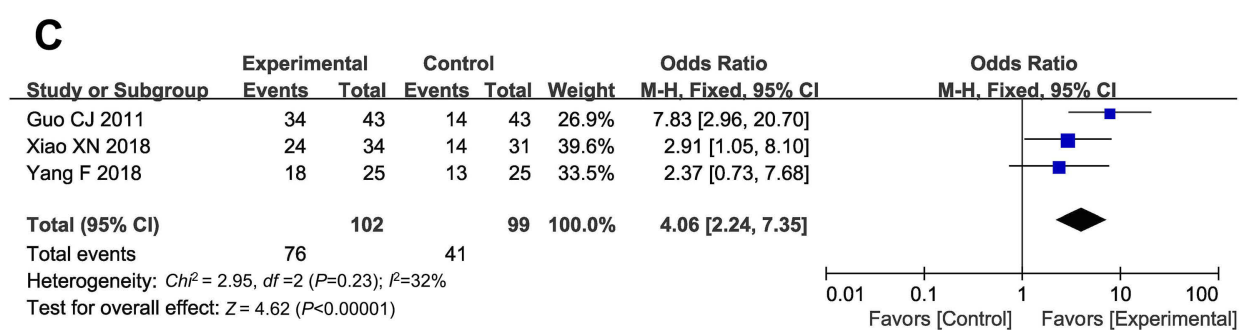

Figure 5 Forest plot of the comparison of quality of life improved rate (QIR, A), karnofsky performance score (KPS, B) and pain relief rate (PRR, C) between the experimental and control group. Control group, chemotherapy alone group; Experimental group, chemotherapy and cinobufotalin combined group. The fixed-effects metaanalysis model (Mantel-Haenszel method) was used.

reliability of our primary conclusions, except those based on a few number of trials.

\section{Sensitivity analysis}

We performed subgroup analysis to explore the source of heterogeneity in ORR and DCR with respect to therapeutic regimens, drug forms of cinobufotalin, sample sizes and research types. As shown in Table 6, our analysis results showed that no significant difference was found between different forms of cinobufotalin, sample sizes and research types. Moreover, cinobufotalin combined with FOLFOX/ $\mathrm{XELOX/capecitabine} \mathrm{chemotherapy} \mathrm{regimens} \mathrm{was} \mathrm{found}$ to be more effective for $\mathrm{GC}$ treatment.

\section{Discussion}

In view of the limitations of the current chemotherapy for malignancies such as drug resistance and toxic side effects, clinicians have been exploring complementary and alternative medicine treatments to improve patients' survival time or QoL and reduce side effects caused by chemotherapy. ${ }^{6,10,46,47}$ Traditional Chinese medicine, particularly cinobufotalin, has been clinically applied as an adjuvant therapy for decades. ${ }^{7,10,11}$ Several studies have been reported that the addition of cinobufotalin could be beneficial to advanced GC patients. ${ }^{16-42}$ Even though there was a statistical analysis of published clinical trials, the exact therapeutic effects were still not systematically evaluated because of small sample sizes and different applied protocols in different studies. Therefore, in this analysis, we conducted a wide range of online search according to strict inclusion and exclusion criteria to provide clear and systematical conclusion.

Our meta-analysis revealed that cinobufotalin and chemotherapy combined therapy for GC patients achieved 


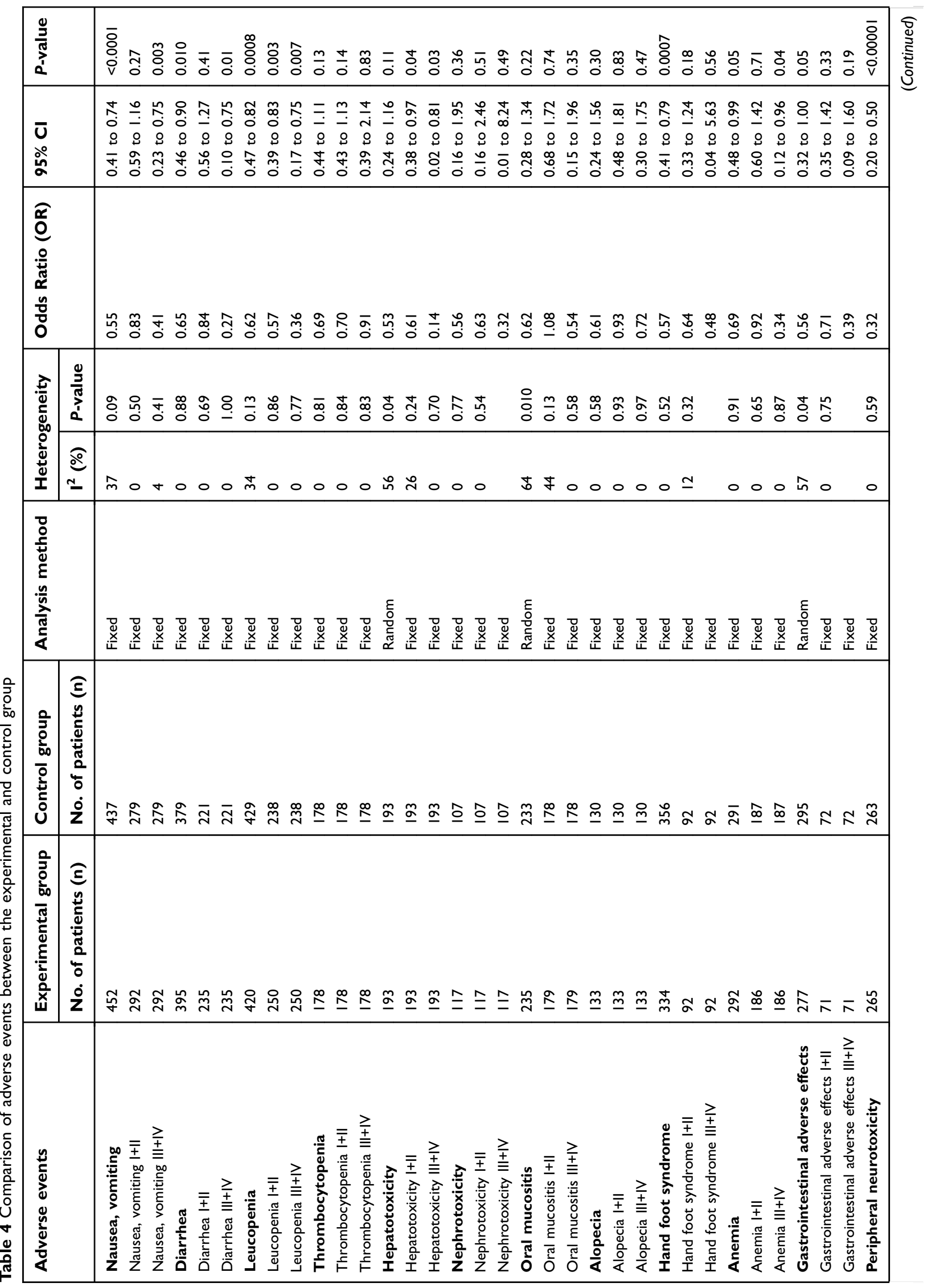




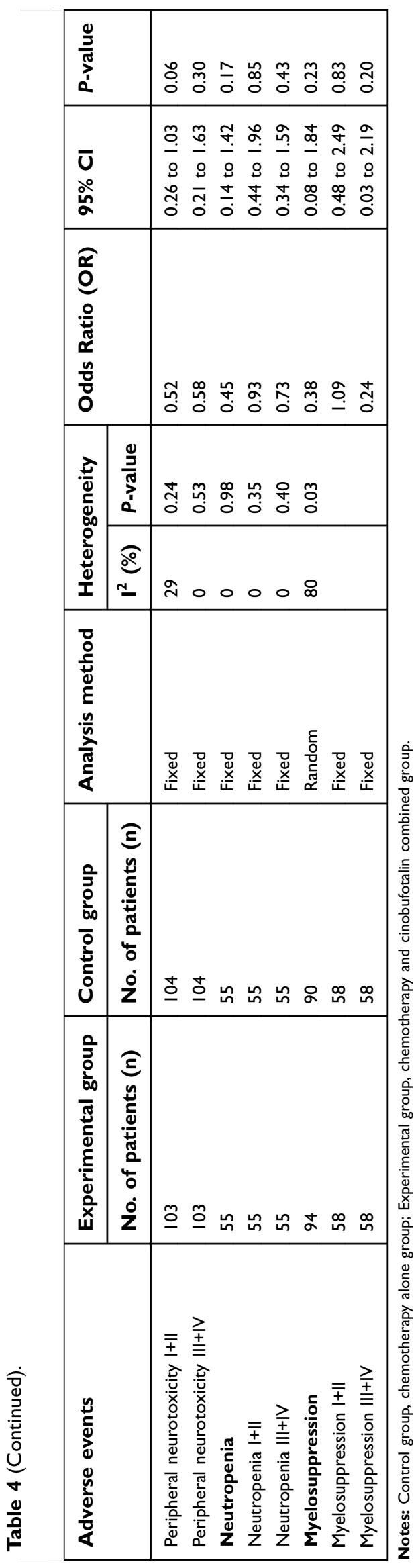

more beneficial effects in comparison with those treated with chemotherapy alone. Combined therapy-treated patients broadly exhibited increased ORR and DCR $(P<0.05)$ and also significantly improved their QoL. These results indicated that using cinobufotalin could improve the curative effects of chemotherapy.

Safety is the top priority of the clinical treatment. One trial $^{7}$ that was conducted at Fudan University Cancer Hospital showed that cinobufotalin is well tolerated by hepatocellular carcinoma, non-small-cell lung cancer and pancreatic cancer patients (only mild adverse events were observed in cancer patients who received cinobufotalin therapy; no grade III and IV toxicities were observed). Our analysis showed that most of the adverse events caused by chemotherapy, including nausea and vomiting, diarrhea, leucopenia, hand-foot syndrome, anemia, gastrointestinal side effects and peripheral neurotoxicity, were alleviated with cinobufotalin combination therapy $(P<0.05)$. Therefore, cinobufotalin is a safe auxiliary antitumor medicine for GC and can effectively alleviate the adverse events associated with chemotherapy.

The analysis on therapeutic effects may be influenced by several factors. In our study, no difference was found between different drug forms of cinobufotalin, sample sizes and research types. Cinobufotalin combined with FOLFOX/ $\mathrm{XELOX/capecitabine} \mathrm{chemotherapy} \mathrm{regimens} \mathrm{was} \mathrm{more}$ effective for GC treatment (Table 6). However, a comparative analysis of the above-mentioned individual chemotherapy regimens should be performed in the future to rule out the possibility that the therapeutic advantage of cinobufotalin combined with FOLFOX, XELOX or capecitabine is due to the better therapeutic effect of them alone compared to that of EOF. As a summary, recent studies on the impact of these factors on the curative effects of cinobufotalin adjuvant therapy remain insufficient, and hence, further investigations should be performed.

There are some limitations in our analysis. First, although traditional Chinese medicine has been exported to 185 countries and regions, its main markets still remained in Asia. ${ }^{48}$ As a traditional medicine, cinobufotalin was mainly applied in China, which may bring the unavoidable regional bias and subsequently influence the clinical application of cinobufotalin worldwide. Second, according to the Cochrane Handbook for systematic reviews of interventions, the most appropriate way of summarizing survival outcomes is to use methods of survival analysis and express the intervention effect as a hazard ratio (HR) because this method takes into 

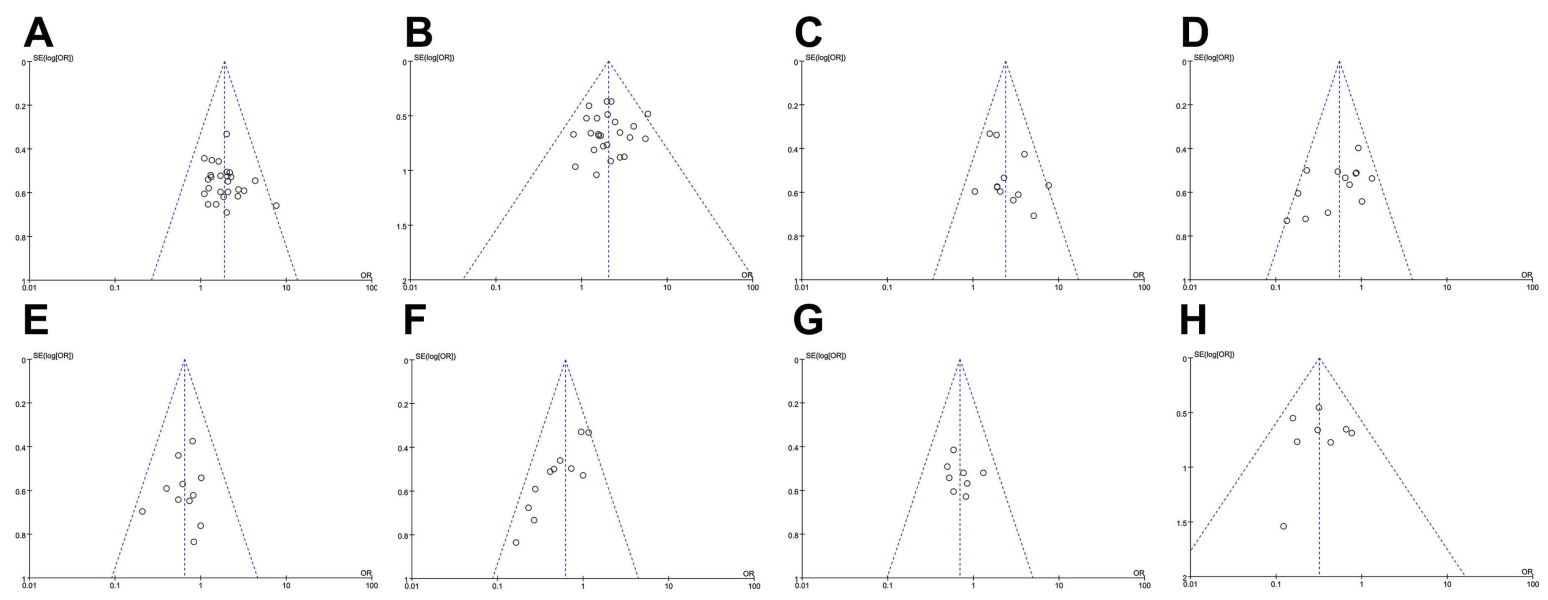

Figure 6 Funnel plot of overall response rate (ORR, A), disease control rate (DCR, B), quality of life improved rate (QIR, C), Nausea and vomiting (D), Diarrhea (E), Leukopenia (F), Anemia (G) and neurotoxicity $(\mathbf{H})$.

Table 5 Publication bias on therapeutic efficacy indexes (CR, PR, SD, PD, ORR, DCR and QIR) and adverse events indexes (Nausea and vomiting, Diarrhea, Leucopenia, Anemia and Neurotoxicity)

\begin{tabular}{|c|c|c|c|c|c|c|c|c|c|c|c|c|}
\hline \multirow{2}{*}{$\begin{array}{l}\text { Publication } \\
\text { Bias }\end{array}$} & \multicolumn{7}{|c|}{ Therapeutic efficacy } & \multicolumn{5}{|c|}{ Adverse events } \\
\hline & CR & PR & SD & PD & ORR & DCR & QIR & $\begin{array}{l}\text { Nausea and } \\
\text { vomiting }\end{array}$ & Diarrhea & Leucopenia & Anemia & Neurotoxicity \\
\hline Begg & 0.742 & 0.038 & 0.513 & 0.870 & 0.280 & 0.870 & 0.304 & 0.161 & 0.755 & 0.003 & 0.454 & 1.000 \\
\hline Egger & 0.833 & 0.015 & 0.721 & 0.905 & 0.331 & 0.905 & 0.235 & 0.069 & 0.623 & $<0.0001$ & 0.528 & 0.894 \\
\hline
\end{tabular}

Note: Parameters discussed in over 8 papers were conducted bias analyses.

Abbreviations: CR, complete response; PR, partial response; SD, stable disease; PD, progressive disease; ORR, overall response rate; DCR, disease control rate; QIR, quality of life improved rate.

consideration the time factor and censored participants. However, the included articles that reported the OS rate only provided the survival number and the total number of patients at 6 months and 12 months, and none of them provided HR with $95 \%$ CI and Kaplan-Meier survival curves. Therefore, there were insufficient data to perform a statistical analysis using HR, which almost certainly will introduce bias. Third, treatment/medical history is very important in evaluating the efficacy of cinobufotalinmediated therapy. However, our data were partly extracted from published papers rather than from the original patient records; therefore, analytical bias would possibly exist. Moreover, the therapeutic effects of the combined therapy may be influenced by numerous variables such as dosage of cinobufotalin, tumor stage and patient's age. However, based on currently available literature, there are insufficient data to perform more statistical analysis to evaluate the correlation. We will keep following up with upcoming clinical trials to obtain relevant data when available. Finally, the follow-up durations of the included studies were short, and the long-term efficacy of cinobufotalin for advanced GC remains to be further evaluated.

\section{Conclusion}

In summary, this meta-analysis indicated that cinobufotalin and chemotherapy combined therapy was effective in treating advanced GC. Clinical application of cinobufotalin not only evidently improved the therapeutic effects of chemotherapy but also effectively alleviated most of the side effects caused by chemotherapy. However, the longterm efficacy of cinobufotalin-mediated adjuvant therapy for advanced GC still needs methodologically rigorous trials to verify its efficacy.

\section{Acknowledgments}

The risk bias assessment in this study was helped and guided by Dr. Ma J (Statistician, Department of Science and Education, Liaocheng People's Hospital). No funding was received for conducting out this study. 


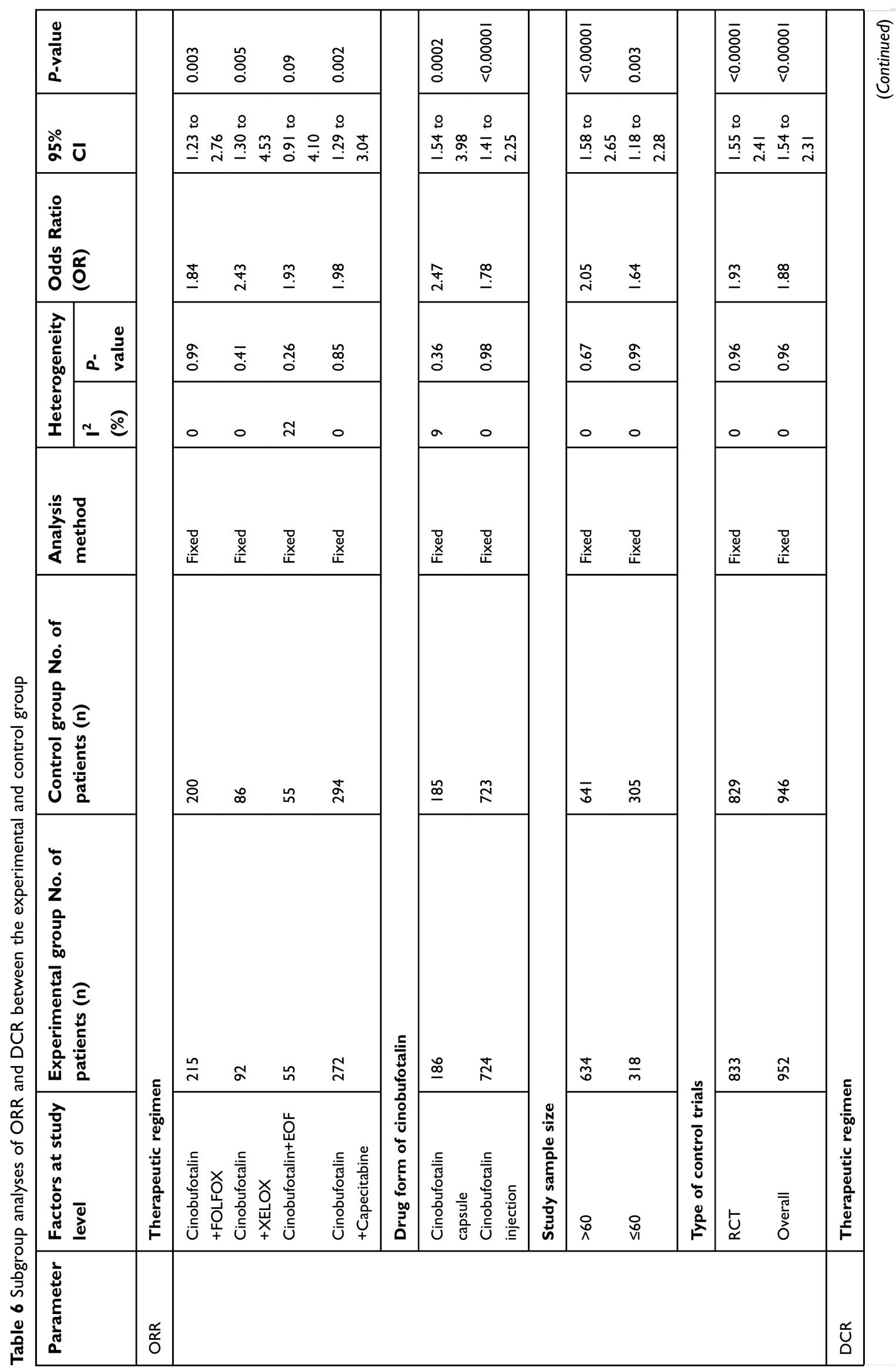




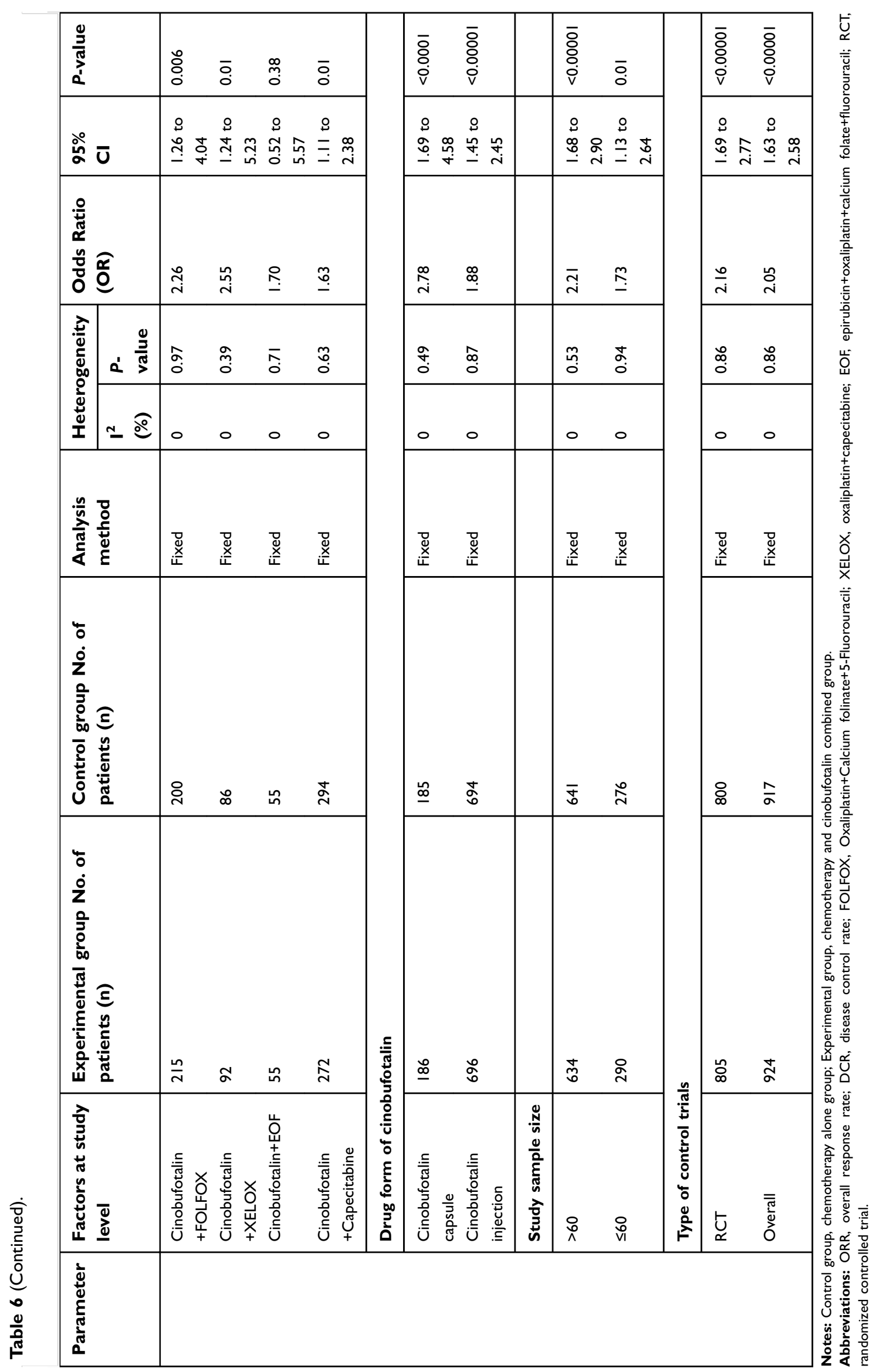




\section{Author contributions}

All authors contributed to study design, data analysis, drafting and revising the article, gave final approval of the version to be published, and agree to be accountable for all aspects of the work.

\section{Disclosure}

The authors report no conflicts of interest in this work.

\section{References}

1. Bray F, Ferlay J, Soerjomataram I, Siegel RL, Torre LA, Jemal A. Global cancer statistics 2018: GLOBOCAN estimates of incidence and mortality worldwide for 36 cancers in 185 countries. CA Cancer J Clin. 2018;68(6):394-424. doi:10.3322/caac.21492

2. Chen W, Zheng R, Baade PD, et al. Cancer statistics in China, 2015. CA Cancer J Clin. 2016;66(2):115-132. doi:10.3322/caac.21338

3. Cheng H, Sun A, Guo Q, Zhang Y. Efficacy and safety of apatinib combined with chemotherapy for the treatment of advanced gastric cancer in the Chinese population: a systematic review and metaanalysis. Drug Des Devel Ther. 2018;12:2173-2183. doi:10.2147/ DDDT.S170678

4. $\mathrm{Mu} \mathrm{Y,} \mathrm{Zhou} \mathrm{CH}$, Chen SF, et al. Effectiveness and safety of chemotherapy combined with cytokine-induced killer cell/dendritic cellcytokine-induced killer cell therapy for treatment of gastric cancer in China: a systematic review and meta-analysis. Cytotherapy. 2016;18 (9):1162-1177. doi:10.1016/j.jcyt.2016.05.015

5. Xue JX, Zhu ZY, Bian WH, Yao C. The traditional Chinese medicine Kangai injection as an adjuvant method in combination with chemotherapy for the treatment of breast cancer in Chinese patients: a meta-analysis. Evid Based Complement Alternat Med. 2018;2018:6305645. doi:10.1155/2018/9567061

6. Chen Y, Zhang G, Chen X, et al. Jianpi Bushen, a traditional Chinese medicine therapy, combined with chemotherapy for gastric cancer treatment: a meta-analysis of randomized controlled trials. Evid Based Complement Alternat Med. 2018;2018:4924279. doi:10.1155/ 2018/9567061

7. Meng Z, Yang P, Shen Y, et al. Pilot study of huachansu in patients with hepatocellular carcinoma, nonsmall-cell lung cancer, or pancreatic cancer. Cancer. 2009;115(22):5309-5318. doi:10.1002/cncr.24602

8. Chen YZ, Feng XB, Li ZD, Zheng WX, Sun H, Li PP. Clinical study on long-term overall survival of advanced non-small-cell lung cancer patients treated with Chinese medicine and Western medicine. Chin J Integr Med. 2014;20(3):179-183. doi:10.1007/s11655-014-1770-6

9. Jiang Y, Liu LS, Shen LP, et al. Traditional Chinese medicine treatment as maintenance therapy in advanced non-small-cell lung cancer: a randomized controlled trial. Complement Ther Med. 2016;24:5562. doi:10.1016/j.ctim.2015.12.006

10. Shi Z, Song T, Wan Y, et al. A systematic review and meta-analysis of traditional insect Chinese medicines combined chemotherapy for non-surgical hepatocellular carcinoma therapy. Sci Rep. 2017;7 (1):4355. doi:10.1038/s41598-017-04351-y

11. Kai S, Lu JH, Hui PP, Zhao H. Pre-clinical evaluation of cinobufotalin as a potential anti-lung cancer agent. Biochem Biophys Res Commun. 2014;452(3):768-774. doi:10.1016/j.bbrc.2014.08.147

12. Emam H, Zhao QL, Furusawa Y, et al. Apoptotic cell death by the novel natural compound, cinobufotalin. Chem Biol Interact. 2012;199 (3):154-160. doi:10.1016/j.cbi.2012.07.005

13. Chen KK, Anderson RC, Henderson FG. Comparison of cardiac action of bufalin, cinobufotalin, and telocinobufagin with cinobufagin. Proc Soc Exp Biol Med. 1951;76(2):372-374.
14. Cheng L, Chen YZ, Peng Y, et al. Ceramide production mediates cinobufotalin-induced growth inhibition and apoptosis in cultured hepatocellular carcinoma cells. Tumour Biol. 2015;36(8):57635771. doi:10.1007/s13277-015-3245-1

15. Li QW, Sun T, Hu KW. Research progress on anti-tumor mechanism of cinobufagin. China J Tradit Chin Med Pharm. 2010;25(12):2075-2078.

16. Cha XT, Hang ZK. Efficacy of cinobufotalin capsule combined with oxaliplatin and tegafur in the treatment of advanced gastric cancer. Psychol Doctor. 2016;22(18):126-127.

17. Chen GF, Jin DX, Li MJ. Clinical observation of cinobufotalin combined with capecitabine in the treatment of 62 cases of senile advanced gastric cancer. Zhejiang J Tradit Chin Med. 2012;47(6):462-463.

18. Chen HM. Efficacy of cinobufotalin combined with TPF regimen in the treatment of advanced gastric cancer. $J$ Emergency Tradit Chin Med. 2009;18(1):35-36.

19. Cui P. Clinical efficacy observation of cinobufotalin in the treatment of advanced gastric cancer. J Liaoning Med Univ. 2009;30(4):333-334.

20. Guo CJ, Yu TH, Zhang HP, Xing JH. The observation of clinical therapeutic effect of cinobufacini combined with docetaxel on advanced stomach cancer. Chin Med Guides. 2011;8(28):54-55.

21. Guo XY, Sun T, Wang XX, Wang Y. Curative efficacy of cinobufacini adjuvant FOLFOX6 regimen in treatment of non operative elderly patients with advanced gastric cancer. J Liaoning Univ Tradit Chin Med. 2013;15(12):190-192.

22. Huang Q, Dong J. Efficacy observation of cinobufotalin injection via hepatic artery perfusion combined with XELOX chemotherapy regimen in the treatment of gastric cancer with liver metastasis. Chin $J$ Tradit Med Sci Technol. 2014;21(3):311-312.

23. Li W, Li HZ. Clinical efficacy analysis of cinobufotalin combined with capecitabine in the treatment of elderly patients with gastric cancer. Med J Chin People'S Health. 2016;28(04):82-83.

24. Li YX. Clinical treatment comparison of 148 elderly patients with advanced gastric cancer. $J$ Front Med. 2012;35:204-205.

25. Lu B, Wu J, Tong RM, Zhang JF. Clinical observation for the combination of capecitabine and cinobufacin capsule on treating elderly advanced gastric cancer. J Liaoning Univ Tradit Chin Med. 2016;18(9):84-87.

26. Lu CH, Hong M, Liu KH, You J. Efficacy observation of neoadjuvant chemotherapy with cinobufotalin in advanced gastric cancer. Tradit Chin Med Jl. 2014;13(3):41-49.

27. Tian B. The enteric capsule of cinobufotalin efficacy combined with chemotherapy in the treatment of gastric cancer. Med Aesthetics Cosmetology. 2012;20(11):33

28. Wang F, Wu LG, Le XY, Chen XD. Clinical effect of gimeracil and oteracil porassium capsules combined with Huachan vegetarian capsules in the treatment of patients with gastric cancer. Chin J Clin Oncol Rehabil. 2014;21(12):1485-1488.

29. Wang WM, Li CF, Yao RJ. Clinical observation of cinobufotalin injection combined with chemotherapy in the treatment of advanced gastric cancer. Clin J Tradit Chin Med. 2010;22(4):314-315.

30. Wang YH. Cinobufotalin injection combined with chemotherapy in the treatment of 36 cases of advanced gastric cancer. Jiangxi $J$ Traditional Chin Med. 2009;40(4):31-32.

31. Wang ZF, Wang P. Efficacy observation of cinobufotalin combined with chemotherapy in the treatment of advanced gastric cancer. Chin J Primary Med Pharm. 2012;19(13):1991-1992.

32. Xiao XN, Lin CH, Li Q, Lin ZJ, Xiao HB. Effect of chemotherapy of DC combined with cinobufacini in the treatment of advanced stomach cancer. Chin J Primary Med Pharm. 2018;25(3):322-324.

33. Xu DM, Liu LJ. Clinical observation of cinobufotalin combined with capecitabine for gastric cancer in elderly patients. Pract $J$ Cancer. 2015;30(3):405-407.

34. Xu YM, Liu S. Efficacy observation of huachansu capsule combined with chemotherapy in treating advanced gastric cancer. World Chin Med. 2016;11(7):1212-1214. 
35. Yang B. Efficacy observation of cinobufotalin injection combined with chemotherapy in the treatment of advanced gastric cancer. World Clin Med. 2017;11(23):92.

36. Yang F, Zhang T. Clinical study of cinobufotalin capsule combined with chemotherapy in the treatment of advanced gastric cancer. Chin Remedies Clin. 2018;18(2):266-268.

37. Zhang CW, Wang QH. Cinobufotalin combined with chemotherapy in the treatment of 35 cases of advanced gastric cancer. J Anhui Tradit Chin Med Coll. 2001;20(4):18-19.

38. Zhang RG, Cheng CH, Shen B, Zhou DM, Zhuang GX. Clinical observation of cinobufotalin combined with chemotherapy in the treatment of advanced gastric cancer. Chin Clin Oncol. 2004;9 (3):269-270

39. Zhang Y, Zhu M, Cao Y, Zhang P, Yao LG, Huang HX. Efficacy observation of cinobufotalin combined with chemotherapy in the treatment of intermediate and late stage gastric cancer. Henan $J$ Oncol. 2005;18(5):359-360.

40. Zheng YL, Ma BH, Yang F. Observation of cinobufotalin combined with chemotherapy for intermediate and late stage gastric cancer. Qingdao Med J. 2007;39(4):260-261.

41. Zhu WK, Li Y, Hou FG, Chen M, Zhou YY. Efficacy of cinobufacini combined with CapeOX regimen in treatment of advanced gastric cancer. Chin Med Guides. 2012;9(5):35-36.

42. Zou HP, Guo XZ, Zhu YF. Clinical research on huachansu with EOF regimen in patients with advanced gastric cancer. Chin J Clin Med. 2012;19(2):140-141.
43. Zeng X, Zhang Y, Kwong JS, et al. The methodological quality assessment tools for preclinical and clinical studies, systematic review and meta-analysis, and clinical practice guideline: a systematic review. J Evid Based Med. 2015;8(1):2-10. doi:10.1111/ jebm. 12141

44. Jackson D, White IR, Riley RD. Quantifying the impact of betweenstudy heterogeneity in multivariate meta-analyses. Stat Med. 2012;31 (29):3805-3820. doi:10.1002/sim.5453

45. Duval S, Tweedie R. Trim and fill: a simple funnel-plot-based method of testing and adjusting for publication bias in meta-analysis. Biometrics. 2000;56(2):455-463.

46. Yan Z, Lai Z, Lin J. Anticancer properties of traditional Chinese medicine. Comb Chem High Throughput Screen. 2017;20(5):423429. doi:10.2174/1386207320666170116141818

47. Xu J, Song Z, Guo Q, Li J. Synergistic effect and molecular mechanisms of traditional Chinese medicine on regulating tumor microenvironment and cancer cells. Biomed Res Int. 2016;2016:1490738. doi:10.1155/2016/1490738

48. Lin AX, Chan $\mathrm{G}, \mathrm{Hu} \mathrm{Y}$, et al. Internationalization of traditional Chinese medicine: current international market, internationalization challenges and prospective suggestions. Chin Med. 2018;13:9. doi:10.1186/s13020-018-0167-z 


\section{Supplementary materials}

A

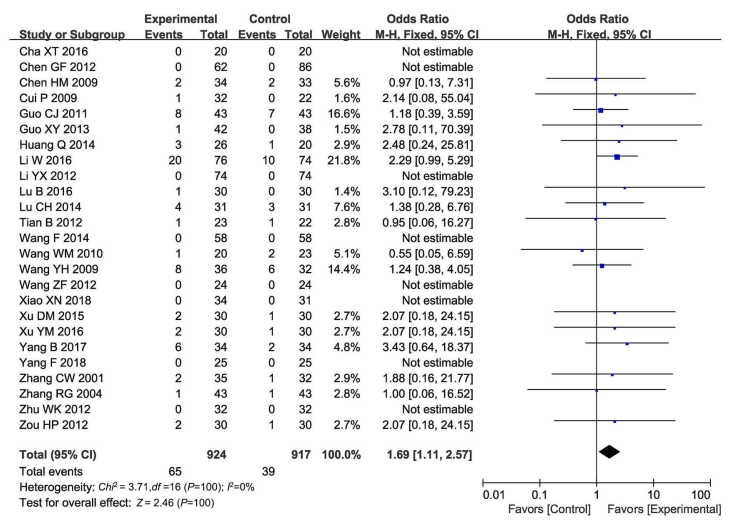

C

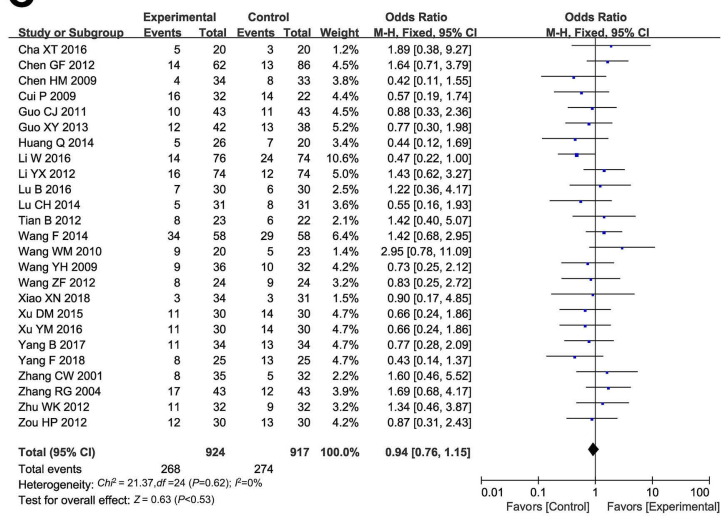

B

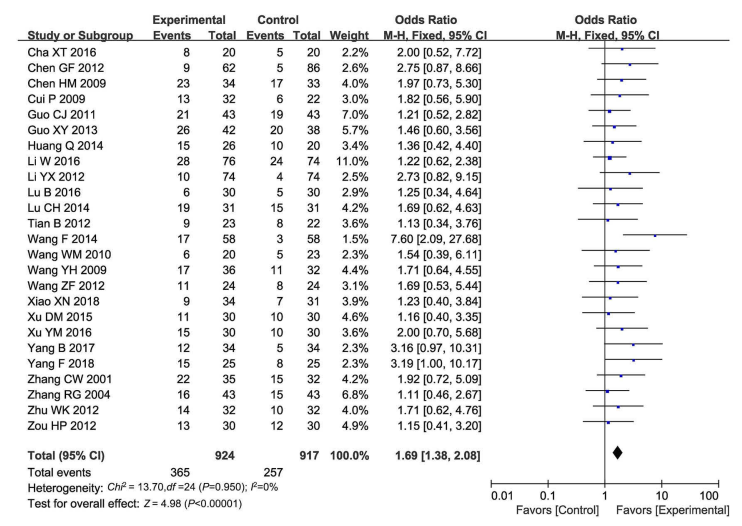

D

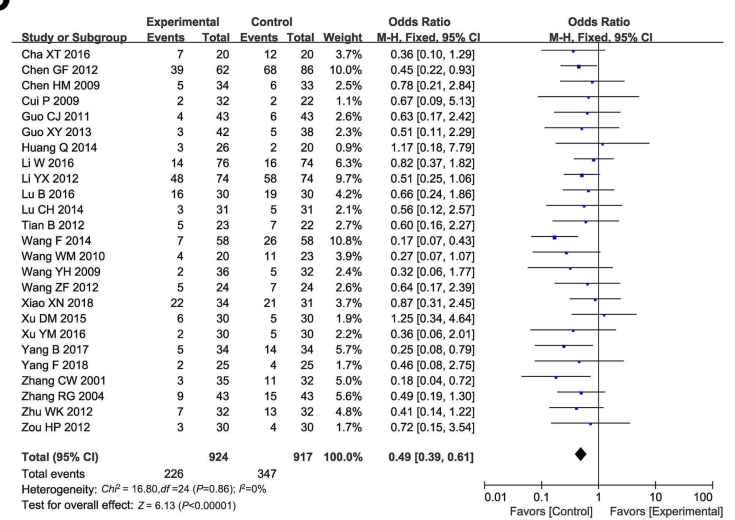

Figure SI Forest plot of the comparison of complete response rates (CR, A), partial response rates (PR, B), stable disease rates (SD, C) and progressive disease rates (PD, D) between the experimental and control group. Control group, chemotherapy alone group; Experimental group, chemotherapy and cinobufotalin combined group. The fixed-effects meta-analysis model (Mantel-Haenszel method) was used. 


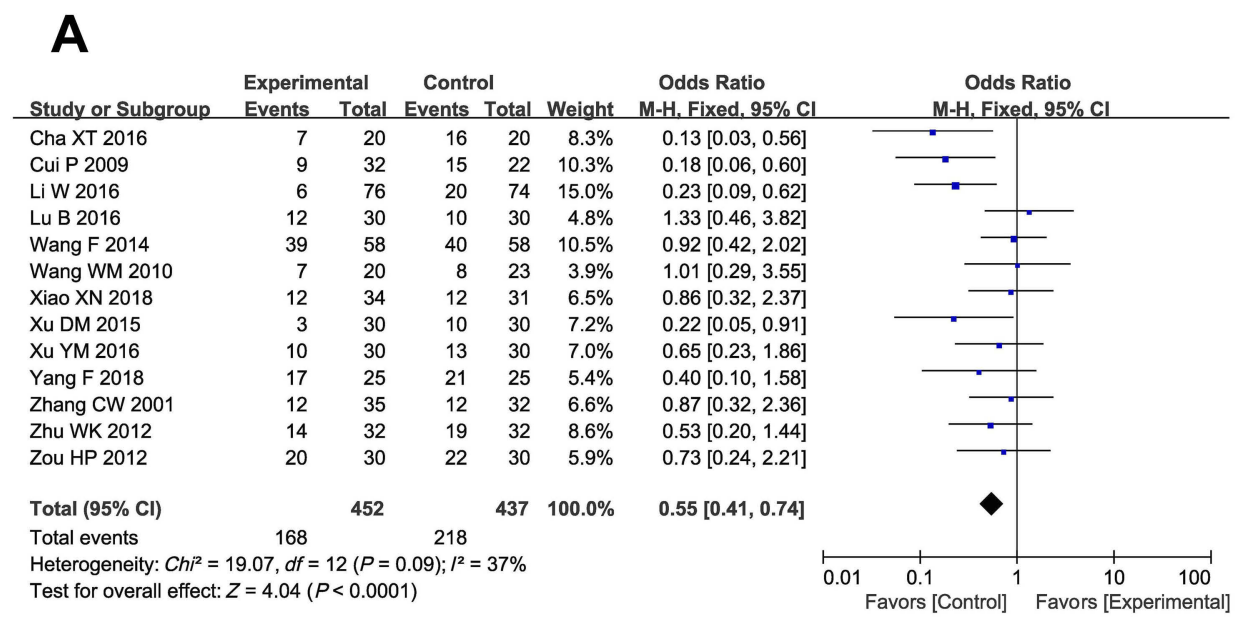

\section{$\mathbf{B}$}

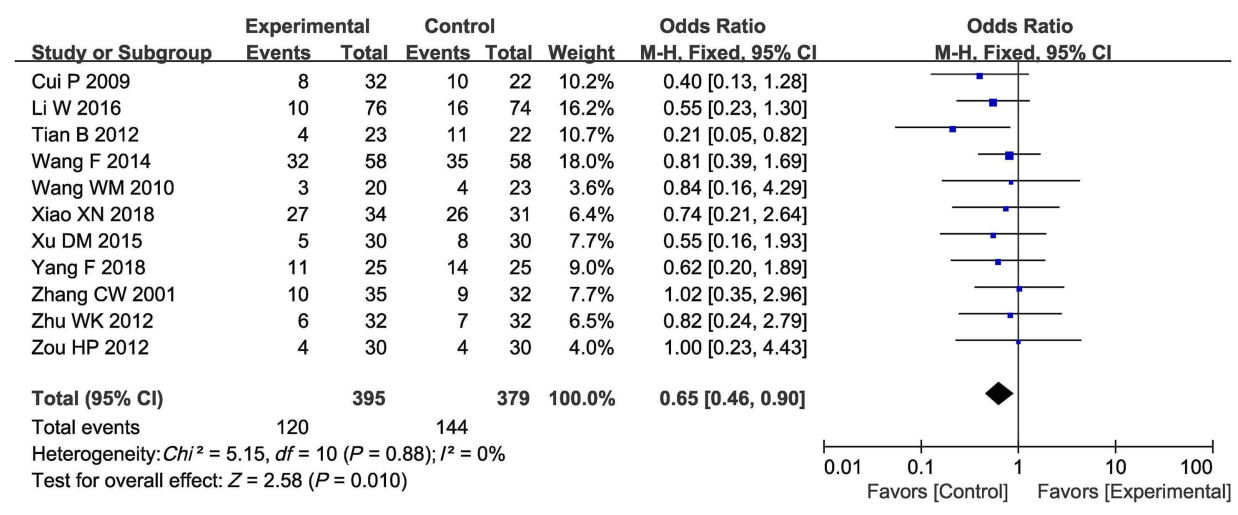

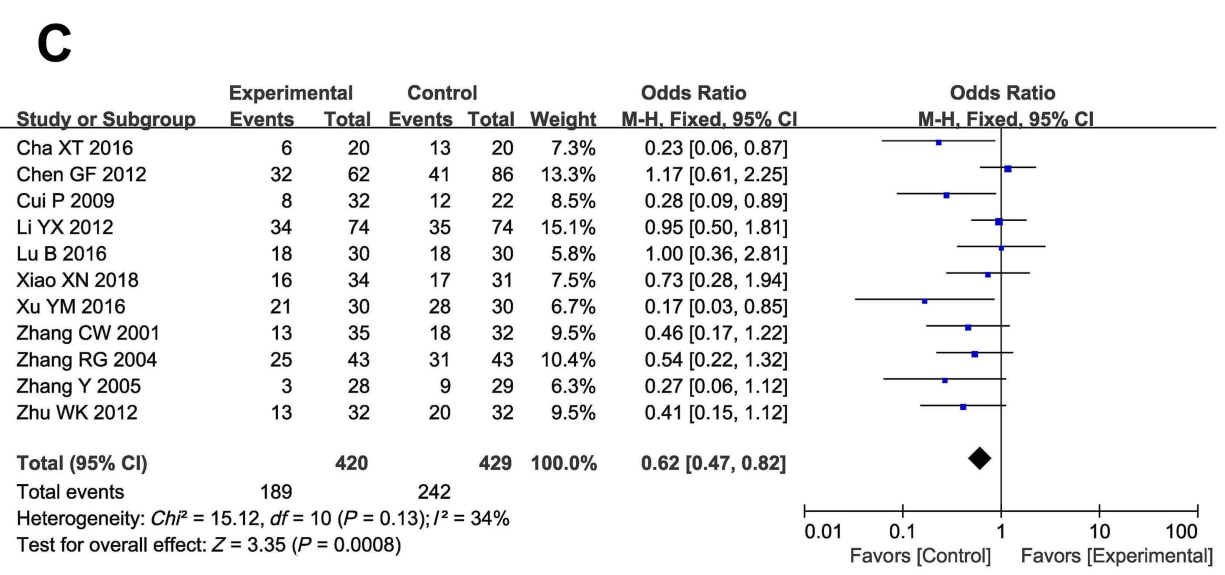

Figure S2 Forest plot of the comparison of adverse effects including nausea and vomiting (A), diarrhea (B), leukopenia (C), thrombocytopenia (D), hepatotoxicity (E), nephrotoxicity $(\mathbf{F})$, oral mucositis $(\mathbf{G})$, alopecia $(\mathbf{H})$, hand-foot syndrome (I), anemia (J), gastrointestinal adverse effects $(\mathbf{K})$, peripheral neurotoxicity $(\mathbf{L})$, neutropenia $(\mathbf{M})$ and myelosuppression $(\mathbf{N})$ between the experimental and control group. Control group, chemotherapy alone group; Experimental group, chemotherapy and cinobufotalin combined group. 


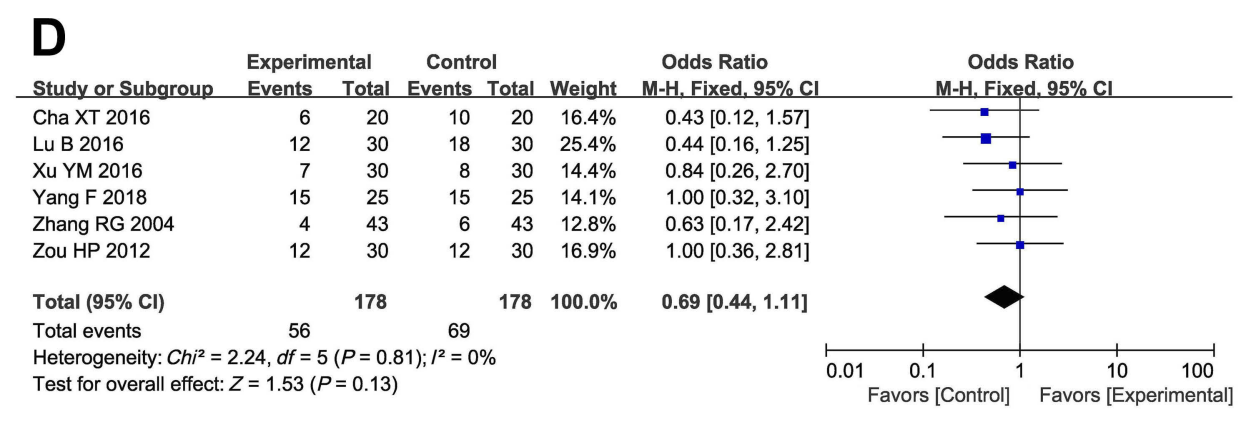

\section{E}

Experimental Control

Odds Ratio

Study or Subgroup Events Total Events Total Weight M-H, Random, 95\% C Cha XT 2016

Lu B 2016

Wang F 2014

Xu YM 2016

Yang F 2018

Zou HP 2012

$\begin{array}{rl}4 & 20 \\ 14 & 30 \\ 23 & 58 \\ 4 & 30 \\ 5 & 25 \\ 1 & 30\end{array}$

$\begin{array}{lll}12 & 20 & 15.7 \%\end{array}$

$30 \quad 20.5 \%$

$\begin{array}{lll}41 & 58 \quad 24.2 \%\end{array}$

$\begin{array}{rrr}41 & 58 & 24.2 \% \\ 4 & 30 & 14.8 \%\end{array}$

$\begin{array}{lll}5 & 25 & 16.0 \%\end{array}$

Total $(95 \% \mathrm{Cl})$

Total events

193

Heterogeneity: $\operatorname{Tau}^{2}=0.51 ; C h i^{2}=11.38, d f=5(P=0.04) ; I^{2}=56 \%$

Test for overall effect: $Z=1.60(P=0.11)$

$0.17[0.04,0.69]$

$1.51[0.54,4.24]$

$0.27[0.13,0.59]$

$1.00[0.23,4.43]$

$1.00[0.25,4.00]$

$0.22[0.02,2.14]$

$0.53[0.24,1.16]$

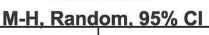

\section{$\mathbf{F}$}

Study or Subgroup Experimental Control $\begin{array}{lllllll}\text { Cui P } 2009 & 2 & 32 & 3 & 22 & 49.9 \% & 0.42[0.06,2.76] \\ X & 2 & 3 & & 30 & 28.06 & 1.00[0.13,7.60]\end{array}$ $\begin{array}{llllll}\text { Xu YM } 2016 & 2 & 30 & 2 & 30 & 28.0 \%\end{array}$

Yang F 2018

Yang F 2018

Zou HP 2012

$\begin{array}{lll}30 & 2 & 30 \\ 25 & 0 & 25\end{array}$

Total $(95 \% \mathrm{Cl})$

Total events

30

$\begin{array}{ll}0 & 25 \\ 1 & 30\end{array}$

$22.1 \%$

117

Heterogeneity: $C h i^{2}=0.51, d f=2(P=0.77) ; I^{2}=0 \%$

Test for overall effect: $Z=0.91(P=0.36)$

$107 \quad 100.0 \%$

Not estimable

$0.32[0.01,8.24]$

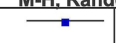

$\mathbf{G}$

Experimental Control

Odds Ratio

Odds Ratio

Study or Subgroup Events Total Events Total Weight M-H, Random, 95\% C

$\begin{array}{llllll}\text { Tian B 2012 } & 2 & 23 & 7 & 22 & 11.0 \%\end{array}$

Wang F 2014

Wang WM 2010

Wang YH 2009

Yang F 2018

Zhang RG 2004

$\begin{array}{rrrrr}40 & 58 & 32 & 58 & 19.0 \% \\ 4 & 20 & 5 & 23 & 12.7 \%\end{array}$

$\begin{array}{rrrrr}4 & 20 & 5 & 23 & 12.7 \% \\ 14 & 15 & 32 & 11.8 \%\end{array}$

$\begin{array}{lll}15 & 25 & 15.6 \%\end{array}$

Zou HP 2012

$\begin{array}{lllll}4 & 43 & 6 & 43 & 13.7 \%\end{array}$

Total $(95 \% \mathrm{Cl})$

Total events

76

$233100.0 \%$

Heterogeneity: $\operatorname{Tau}^{2}=0.68 ; C h i^{2}=16.87, d f=6(P=0.010) ; I^{2}=64 \%$

Test for overall effect: $Z=1.22(P=0.22)$

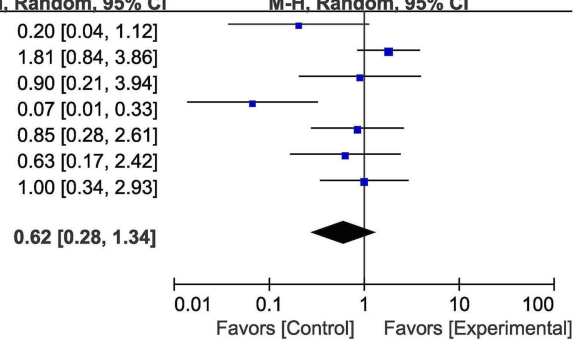

Figure S2 (Continued). 


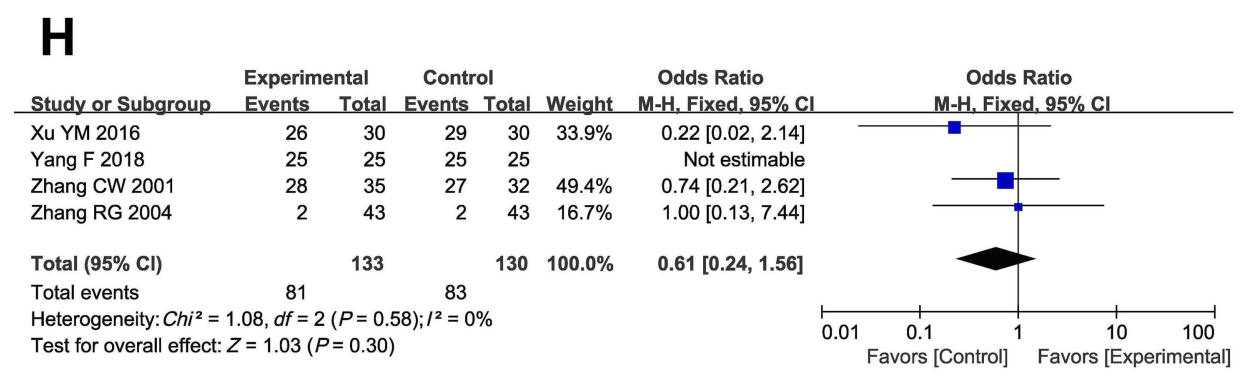

I

\begin{tabular}{|c|c|c|c|c|c|c|c|c|c|}
\hline \multirow{2}{*}{ Study or Subgroup } & \multicolumn{2}{|c|}{ Experimental } & \multicolumn{2}{|c|}{ Control } & \multirow{2}{*}{ Weight } & \multirow{2}{*}{$\begin{array}{l}\text { Odds Ratio } \\
\text { M-H, Fixed, } 95 \% \mathrm{Cl}\end{array}$} & \multirow{2}{*}{\multicolumn{3}{|c|}{$\begin{array}{c}\text { Odds Ratio } \\
\text { M-H, Fixed, } 95 \% \mathrm{Cl}\end{array}$}} \\
\hline & & & & Total & & & & & \\
\hline Chen GF 2012 & 29 & 62 & 48 & 86 & $21.8 \%$ & $0.70[0.36,1.34]$ & $\rightarrow$ & & \\
\hline Li W 2016 & 14 & 76 & 28 & 74 & $23.5 \%$ & $0.37[0.18,0.78]$ & & & \\
\hline Li YX 2012 & 39 & 74 & 44 & 74 & $21.2 \%$ & $0.76[0.40,1.46]$ & $\rightarrow$ & & \\
\hline Lu B 2016 & 18 & 30 & 20 & 30 & $8.1 \%$ & $0.75[0.26,2.15]$ & & & \\
\hline Xu DM 2015 & 7 & 30 & 14 & 30 & $10.9 \%$ & $0.35[0.11,1.05]$ & & & \\
\hline Zhu WK 2012 & 5 & 32 & 5 & 32 & $4.3 \%$ & $1.00[0.26,3.86]$ & & & \\
\hline Zou HP 2012 & 5 & 30 & 12 & 30 & $10.2 \%$ & $0.30[0.09,1.00]$ & & & \\
\hline Total $(95 \% \mathrm{Cl})$ & & 334 & & 356 & $100.0 \%$ & $0.57[0.41,0.79]$ & & & \\
\hline Total events & 117 & & 171 & & & & & & \\
\hline $\begin{array}{l}\text { Heterogeneity: } \text { Chi }^{2}= \\
\text { Test for overall effect }\end{array}$ & $\begin{array}{l}15, d f= \\
=3.40(t\end{array}$ & $\begin{array}{l}(P=0.5 \\
=0.000\end{array}$ & 2); $1^{2}=0$ & & & & $\begin{array}{lcc}0.01 & 0.1 & 1 \\
& \text { Favors [Control] }\end{array}$ & $\begin{array}{lr}1 & 10 \\
& \text { Favors [EX }\end{array}$ & $\begin{array}{l}0 \\
0 \\
\text { Experimental] }\end{array}$ \\
\hline
\end{tabular}

\section{J}

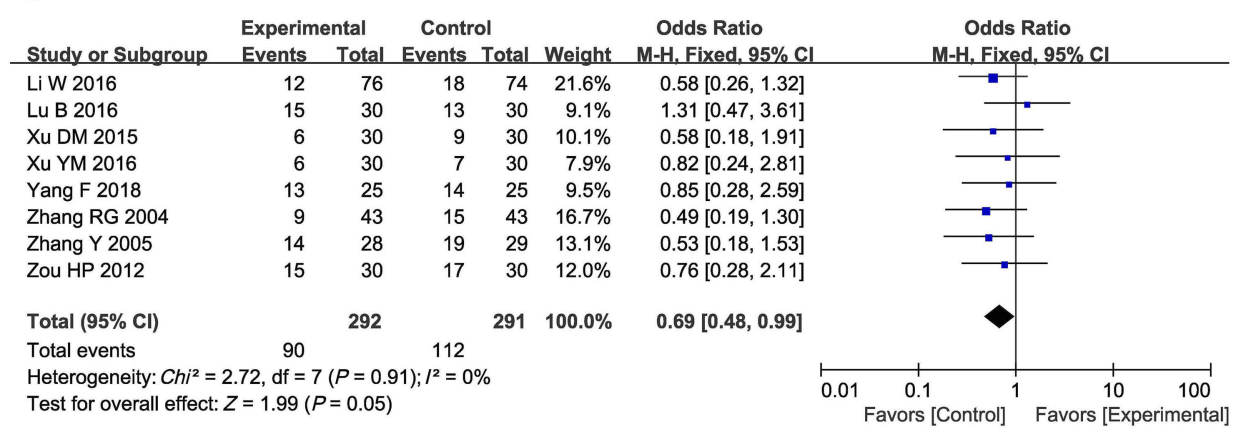

\section{K}

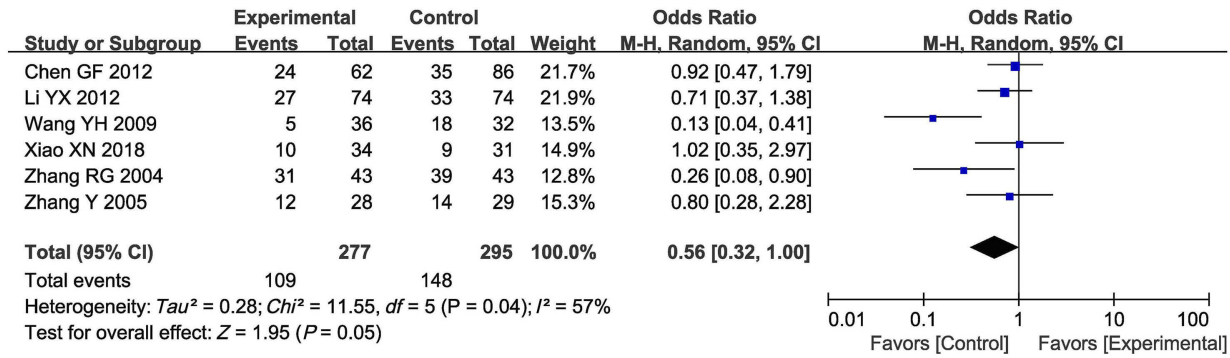

Figure S2 (Continued). 


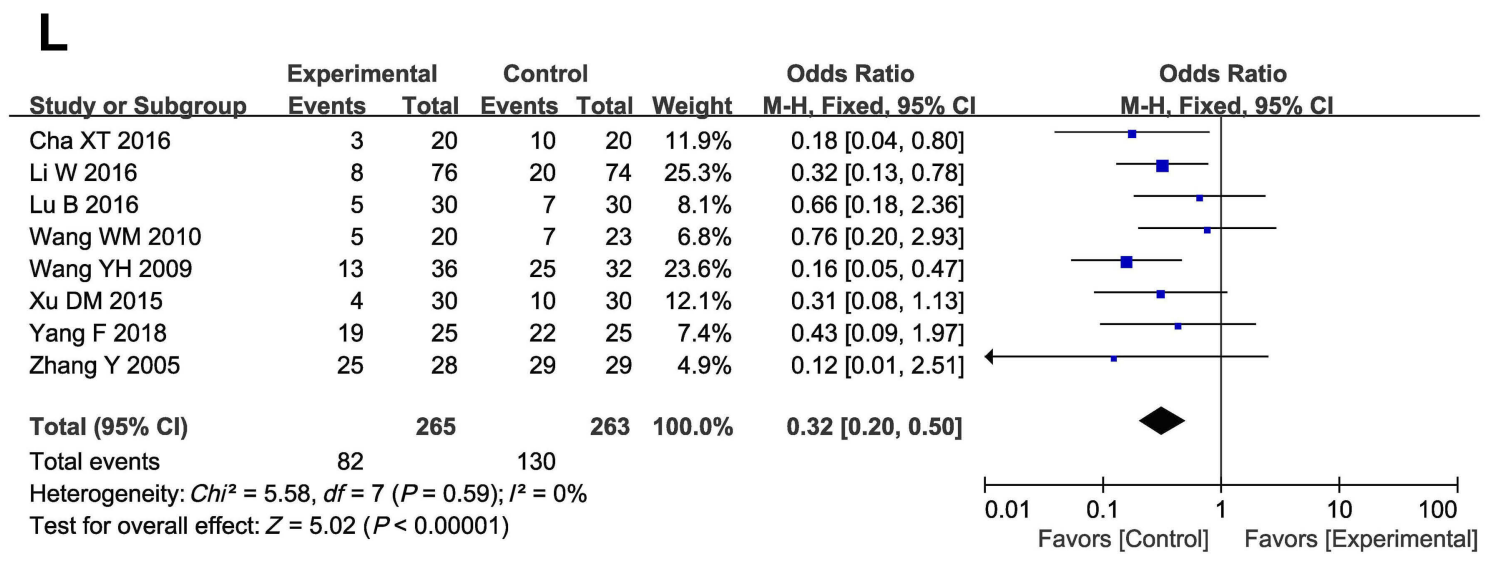

\section{M}

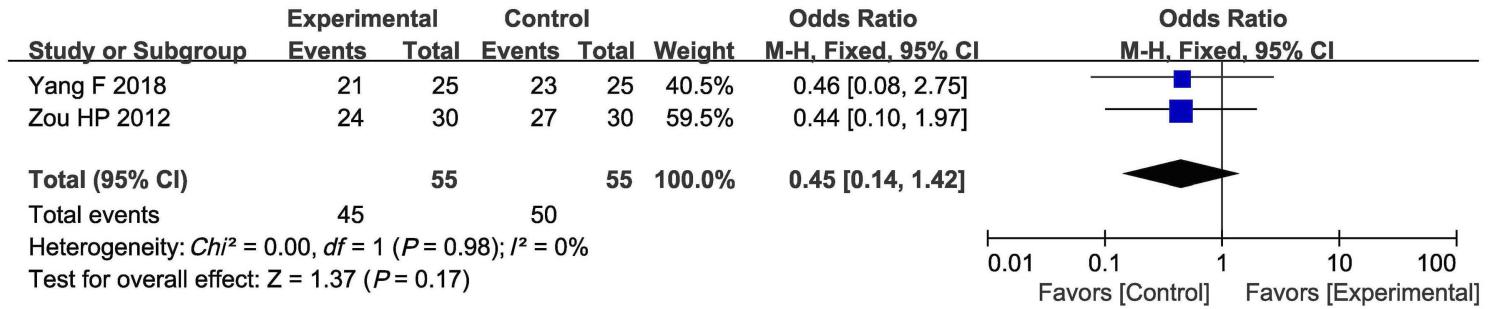

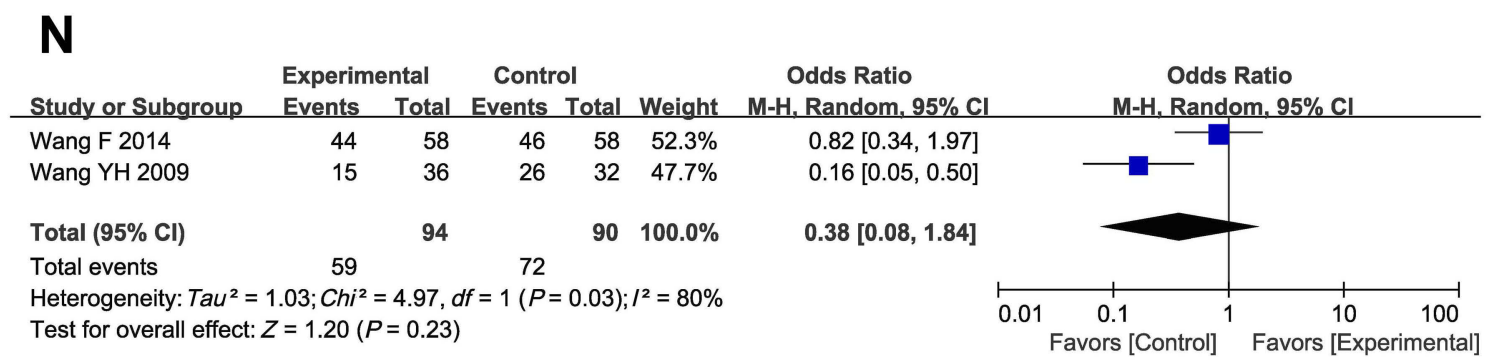

Figure S2 (Continued). 


\section{A}

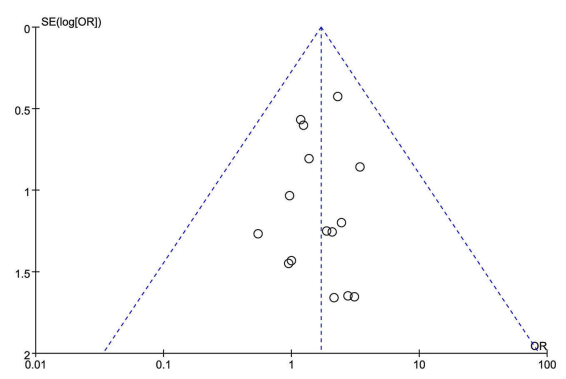

C

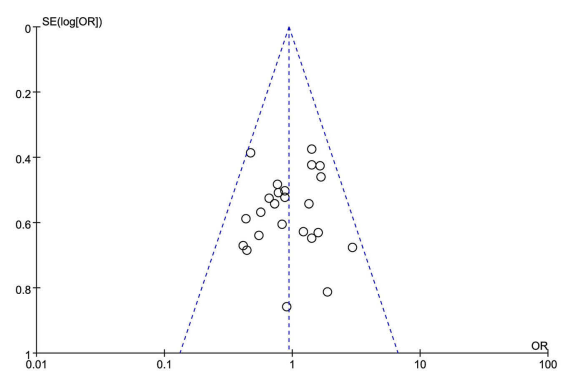

B

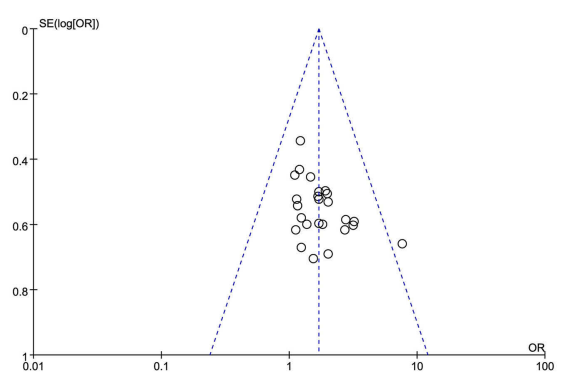

D

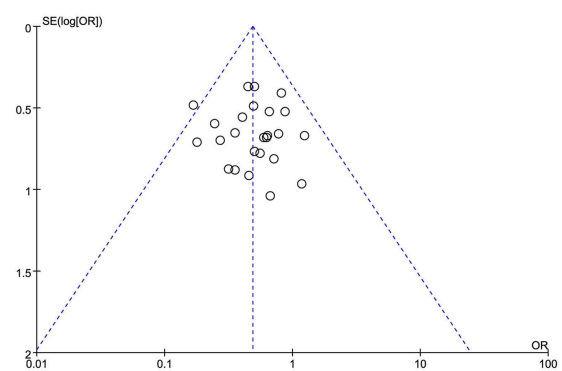

Figure S3 Funnel plot of percentage of complete response rates (CR, A), partial response rates (PR, B), stable disease rates (SD, C) and progressive disease rates (PD, D).

\section{Publish your work in this journal}

OncoTargets and Therapy is an international, peer-reviewed, open access journal focusing on the pathological basis of all cancers, potential targets for therapy and treatment protocols employed to improve the management of cancer patients. The journal also focuses on the impact of management programs and new therapeutic agents and protocols on patient perspectives such as quality of life, adherence and satisfaction. The manuscript management system is completely online and includes a very quick and fair peer-review system, which is all easy to use. Visit http://www.dovepress.com/ testimonials.php to read real quotes from published authors. 